\title{
The maximum average connectivity among all orientations of a graph
}

\author{
Rocío M. Casablanca ${ }^{1}$. Peter Dankelmann ${ }^{2} \cdot$ Wayne Goddard $^{2,3}$. \\ Lucas $\mathrm{Mol}^{4}$ (D) Ortrud Oellermann ${ }^{5}$
}

Accepted: 8 July 2021

(c) The Author(s), under exclusive licence to Springer Science+Business Media, LLC, part of Springer Nature 2021

\begin{abstract}
For distinct vertices $u$ and $v$ in a graph $G$, the connectivity between $u$ and $v$, denoted $\kappa_{G}(u, v)$, is the maximum number of internally disjoint $u-v$ paths in $G$. The average connectivity of $G$, denoted $\bar{\kappa}(G)$, is the average of $\kappa_{G}(u, v)$ taken over all unordered pairs of distinct vertices $u, v$ of $G$. Analogously, for a directed graph $D$, the connectivity from $u$ to $v$, denoted $\kappa_{D}(u, v)$, is the maximum number of internally disjoint directed $u-v$ paths in $D$. The average connectivity of $D$, denoted $\bar{\kappa}(D)$, is the average of $\kappa_{D}(u, v)$ taken over all ordered pairs of distinct vertices $u, v$ of $D$. An orientation of a graph $G$ is a directed graph obtained by assigning a direction to every edge of $G$. For a graph $G$, let $\bar{\kappa}_{\max }(G)$ denote the maximum average connectivity among all orientations of $G$. In this paper we obtain bounds for $\bar{\kappa}_{\max }(G)$ and for the ratio $\bar{\kappa}_{\max }(G) / \bar{\kappa}(G)$ for
\end{abstract}

Ortrud Oellermann supported by an NSERC Grant CANADA, Grant number RGPIN-2016-05237. Lucas Mol supported by an NSERC Grant CANADA, Grant number RGPIN-2021-04084.

$凶$ Lucas Mol

1mol@tru.ca

Rocío M. Casablanca

rociomc@us.es

Peter Dankelmann

pdankelmann@uj.ac.za

Wayne Goddard

goddard@clemson.edu

Ortrud Oellermann

o.oellermann@uwinnipeg.ca

1 Universidad de Sevilla, Seville, Spain

2 University of Johannesburg, Johannesburg, South Africa

3 Clemson University, Clemson, USA

4 Thompson Rivers University, Kamloops, Canada

5 University of Winnipeg, Winnipeg, Canada

Published online: 12 August 2021 
all graphs $G$ of a given order and in a given class of graphs. Whenever possible, we demonstrate sharpness of these bounds. This problem had previously been studied for trees. We focus on the classes of cubic 3-connected graphs, minimally 2-connected graphs, 2-trees, and maximal outerplanar graphs.

Keywords Connectivity · Average connectivity · Orientations · Cubic graphs · Minimally 2-connected graphs · Maximal outerplanar graphs

\section{Introduction}

In this article, a graph is finite, loopless, and contains no multiple edges. An orientation of a graph $G$ is a directed graph obtained by assigning a direction to every edge of $G$. Connectedness properties of orientations of graphs have been studied in a variety of different settings. Probably the most well-known and oldest result in this area is Robbins' Theorem (Robbins 1939), which states that every 2-edge-connected graph has a strong orientation, i.e., an orientation with the property that for every pair $u, v$ of distinct vertices of the graph, there is both a directed $u-v$ path and a directed $v-u$ path. Nash-Williams (1960) extended this result by showing that every $2 k$-edge-connected graph has a strongly $k$-edge-connected orientation, i.e., an orientation for which there exist $k$ edge-disjoint paths from $u$ to $v$ for every ordered pair $(u, v)$ of distinct vertices of the graph. Mader (1978) also established this result using his so-called lifting theorem. In light of these results on edge connectivity, it is natural to ask what can be said about the connectivity of an orientation of a graph in terms of its connectivity. Thomassen (1989) proposed the following conjecture.

Conjecture 1.1 For every positive integer $k$, there exists a smallest positive integer $f(k)$ such that every $f(k)$-connected graph has a strongly $k$-connected orientation.

More recently, Thomassen (2014) established the following necessary and sufficient conditions that guarantee that a graph admits a strongly 2-connected orientation.

Theorem 1.2 (Thomassen 2014) A graph G has a strongly 2-connected orientation if and only if $G$ is 4-edge-connected, and every vertex-deleted subgraph of $G$ is 2-edgeconnected.

It follows that if $G$ is 4-connected, then $G$ has a strongly 2-connected orientation, confirming Conjecture 1.1 in the case $k=2$. Conjecture 1.1 remains open for $k \geq 3$. Durand de Gevigney (2020) showed that for each $k \geq 3$, the problem of deciding whether a given graph has a strongly $k$-connected orientation is NP-complete.

Instead of trying to find the largest $k$ for which a given graph has a strongly $k$-connected orientation, we focus on finding orientations for which the average connectivity, that is, the average of the connectivities between all ordered pairs of vertices, is maximized.

For distinct vertices $u$ and $v$ in a graph $G$, the connectivity between $u$ and $v$, denoted $\kappa_{G}(u, v)$, or $\kappa(u, v)$ if $G$ is clear from context, is the maximum number of internally disjoint $u-v$ paths in $G$. If $u$ and $v$ are not adjacent in $G$, then Menger's Theorem 
states that $\kappa_{G}(u, v)$ is also equal to the minimum number of vertices whose removal separates $u$ and $v$ in $G$. It is also well-known that the connectivity $\kappa(G)$ of a graph $G$ is the minimum of $\kappa_{G}(u, v)$ taken over all unordered pairs of distinct vertices $u, v$ of $G$. See Oellermann (2013) for more details.

The average connectivity of $G$, denoted $\bar{\kappa}(G)$, is the average of $\kappa_{G}(u, v)$ taken over all unordered pairs of distinct vertices $u, v$ of $G$. If $G$ has order $n$, then

$$
\bar{\kappa}(G)=\frac{1}{\left(\begin{array}{c}
n \\
2
\end{array}\right)} \sum_{\{u, v\} \subseteq V(G)} \kappa_{G}(u, v) .
$$

This parameter was introduced by Beineke et al. (2002) as a more refined measure of the connectedness of a graph than the connectivity. Bounds on the average connectivity of a graph in terms of various graph parameters were given by Dankelmann and Oellermann (2003).

For a directed graph $D$ with distinct vertices $u$ and $v$, the connectivity from $u$ to $v$, denoted $\kappa_{D}(u, v)$, is the maximum number of internally disjoint directed $u-v$ paths in $D$. The average connectivity of $D$, denoted $\bar{\kappa}(D)$, is defined as the average of $\kappa_{D}(u, v)$ taken over all ordered pairs of distinct vertices $u, v$ of $D$. If $D$ has order $n$, then

$$
\bar{\kappa}(D)=\frac{1}{n(n-1)} \sum_{\substack{(u, v) \\ u, v \in V(D), u \neq v}} \kappa_{D}(u, v) .
$$

The average connectivity of digraphs was first introduced by Henning and Oellermann (2004).

In this article, we are concerned primarily with the maximum average connectivity among all orientations of a given graph $G$, denoted $\bar{\kappa}_{\max }(G)$. This parameter was also introduced by Henning and Oellermann (2004). If $D$ is an orientation of $G$ such that $\bar{\kappa}_{\max }(G)=\bar{\kappa}(D)$, then we say that $D$ is an optimal orientation of $G$. Note that optimal orientations need not be unique; a graph may have many different optimal orientations. Henning and Oellermann gave the following asymptotically sharp bound on $\bar{\kappa}_{\max }(T)$ for any tree $T .^{1}$

1 The family of trees described in Henning and Oellermann (2004) for which the lower bound is asymptotically sharp can be obtained as follows. For a given $t \geq 1$, take three copies of $K_{1, t}$, and identify a leaf from each copy of $K_{1, t}$ in a single vertex (which will have degree 3). Let $T_{3 t+1}$ be such a tree. So if $n=3 t+1$, then $\bar{\kappa}_{\max }\left(T_{3 t+1}\right)=\frac{2 n^{2}+14 n-43}{9 n(n-1)}$. We point out that it was incorrectly stated in Henning and Oellermann (2004) that for a tree $T$ of order $n \geq 3$, we have $\bar{\kappa}_{\max }(T) \geq \frac{2 n^{2}+14 n-43}{9 n(n-1)}$. This inequality holds for $n \geq 34$. However, for $n<34$, we have

$$
\bar{\kappa}_{\max }\left(K_{1, n-1}\right)=\frac{\left\lfloor\frac{n-1}{2}\right\rfloor\left\lceil\frac{n-1}{2}\right\rceil+(n-1)}{n(n-1)}<\frac{2 n^{2}+14 n-43}{9 n(n-1)} .
$$

For $n<34$, the stars are in fact the extremal trees. That is, for $n<34$, one can show that $\bar{\kappa}_{\text {max }}\left(K_{1, n-1}\right) \leq$ $\bar{\kappa}_{\max }(T)$ for every tree $T$ of order $n$. 
Theorem 1.3 (Henning and Oellermann 2004) If $T$ is a tree of order $n \geq 3$, then

$$
\frac{2}{9}<\bar{\kappa}_{\max }(T) \leq \frac{1}{2}
$$

We obtain bounds on $\bar{\kappa}_{\max }(G)$ for all graphs $G$ of a given order and belonging to a given class of graphs. Whenever possible, we demonstrate that these bounds are (asymptotically) sharp. We study these problems for two classes of graphs that are, in some sense, generalizations or extensions of trees: minimally 2-connected graphs (trees are minimally 1-connected), and maximal outerplanar graphs (which are known to be 2-trees).

We are also interested in the value of the ratio $\bar{\kappa}_{\max }(G) / \bar{\kappa}(G)$ for a given graph $G$, which is in some sense a measure of how well the overall level of connectedness of a graph can be preserved under orientation. Naively, one might expect the ratio $\bar{\kappa}_{\max }(G) / \bar{\kappa}(G)$ to be close to $1 / 2$, since one might hope for an orientation of $G$ in which a collection of $\kappa_{G}(u, v)$ internally disjoint paths are all directed one way or the other, for every pair of distinct vertices $u, v$. But the directed $u-v$ paths need not be internally disjoint from the directed $v-u$ paths, meaning that $\bar{\kappa}_{\max }(G) / \bar{\kappa}(G)$ can be much larger than $1 / 2$; we show that it can be arbitrarily close to 1 . It is also true that $\bar{\kappa}_{\max }(G) / \bar{\kappa}(G)$ can be much smaller than $1 / 2$. For example, we have already mentioned that there are trees $T$ such that $\bar{\kappa}_{\text {max }}(T)$ is arbitrarily close to $2 / 9$. Since $\bar{\kappa}(T)=1$ for every tree $T$, it follows immediately that $\bar{\kappa}_{\max }(T) / \bar{\kappa}(T)$ can be arbitrarily close to $2 / 9$.

We now describe our main contributions, and the layout of the article. In Sect. 2, we present some terminology, and some straightforward bounds on $\bar{\kappa}_{\max }(G)$ and $\bar{\kappa}_{\max }(G) / \bar{\kappa}(G)$ for every graph $G$. We briefly consider the edge connectivity analogue of $\bar{\kappa}_{\max }(G) / \bar{\kappa}(G)$ in order to highlight a stark contrast between connectivity and edge connectivity in this setting.

In Sect. 3, we show that if $G$ is an $r$-regular graph of order $n$ for odd $r$, then

$$
\bar{\kappa}_{\max }(G) \leq \frac{r-1}{2}+\frac{n}{4(n-1)},
$$

and that this bound is sharp. We then focus on cubic 3-connected graphs. If $G$ is a cubic 3 -connected graph, then certainly $\bar{\kappa}_{\max }(G) \geq 1$ by Robbins' Theorem. We demonstrate that this lower bound is asymptotically tight by describing a sequence of cubic 3-connected graphs for which the values of $\bar{\kappa}_{\max }$ approach 1 . This shows that one cannot guarantee significantly more 'connectedness' in an optimal orientation of a 3-connected graph than in an optimal orientation of a 2-edge-connected graph.

In Sect. 4 , we show that if $G$ is a minimally 2-connected graph of order $n$, then

$$
1 \leq \bar{\kappa}_{\max }(G)<\frac{5}{4}
$$

While the lower bound is sharp, we suspect that the upper bound can be improved. We also show that for every minimally 2 -connected graph $G$,

$$
\frac{4}{9}<\frac{\bar{\kappa}_{\max }(G)}{\bar{\kappa}(G)}<\frac{5}{8}
$$


Although we are unable to show that these bounds are sharp, we do find sequences of minimally 2-connected graphs for which the ratio $\bar{\kappa}_{\max }(G) / \bar{\kappa}(G)$ approaches $\frac{25}{54}$ and $\frac{9}{16}$, respectively. One of these constructions uses the sequence of cubic 3-connected graphs described in Sect. 3.

Finally, in Sect. 5, we show that if $G$ is a maximal outerplanar graph, then

$$
\bar{\kappa}_{\max }(G) \leq \frac{3}{2}+\frac{n-5}{n^{2}-n},
$$

and that this bound is asymptotically sharp. We conjecture that if $G$ is a maximal outerplanar graph of order at least 4 , then $\bar{\kappa}_{\max }(G) \geq \frac{19}{18}$. We give an example which demonstrates that if this conjecture is true, then the bound is asymptotically sharp.

\section{General bounds}

We begin with some notation and terminology. The total connectivity of a graph $G$ is the sum of the connectivities of all unordered pairs of distinct vertices of $G$, and is denoted by $K(G)$. Evidently, if $G$ has order $n$, then $K(G)=\left(\begin{array}{c}n \\ 2\end{array}\right) \bar{\kappa}(G)$. If $u$ and $v$ are distinct vertices of $G$, then $\kappa(u, v) \leq \min \{\operatorname{deg}(u)+\operatorname{deg}(v)\}$. Thus, if $d_{1}, d_{2}, \ldots, d_{n}$ is the degree sequence of $G$, then $K(G) \leq \sum_{1 \leq i<j \leq n} \min \left\{d_{i}, d_{j}\right\}$. In this case, we call

$$
P(G)=P\left(d_{1}, d_{2}, \ldots, d_{n}\right)=\sum_{1 \leq i<j \leq n} \min \left\{d_{i}, d_{j}\right\}
$$

the potential of $G$.

If $D$ is a digraph, and $u, v \in V(D)$ are distinct, then we let $\theta_{D}(u, v)=\kappa_{D}(u, v)+$ $\kappa_{D}(v, u)$. If $D$ is clear from context, then the subscript will be omitted. We also refer to $\theta(u, v)$ as the $\theta$ value for $u$ and $v$. For every pair $u, v$ of distinct vertices of $D$, we have $\theta(u, v)=\kappa(u, v)+\kappa(v, u) \leq \min \{\operatorname{od}(u), \operatorname{id}(v)\}+\min \{\operatorname{od}(v), \operatorname{id}(u)\}$. We call

$$
P(D)=\sum_{\{u, v\} \subseteq V(D)}(\min \{\operatorname{od}(u), \operatorname{id}(v)\}+\min \{\operatorname{od}(v), \operatorname{id}(u)\})
$$

the potential of $D$.

The total connectivity of a digraph $D$, denoted $K(D)$, is the sum of the connectivities of all ordered pairs of distinct vertices of $D$, or equivalently, the sum of the $\theta$ values of all unordered pairs of distinct vertices of $D$. If $D$ has order $n$, then $K(D)=$ $n(n-1) \bar{\kappa}(D)$.

For a graph $G$, the notation $K_{\max }(G)$ denotes the maximum total connectivity among all orientations of $G$. The potential of $G$ provides the following useful upper bound on the total connectivity of any orientation of $G$, and hence on $K_{\max }(G)$.

Observation 2.1 If $D$ is an orientation of a graph $G$, then

$$
K(D)=\sum_{\{u, v\} \subseteq V(D)} \theta(u, v) \leq P(D) \leq P(G) .
$$


Fig. 1 The orientation $D_{2 n}$ of the graph $F_{2 n}$

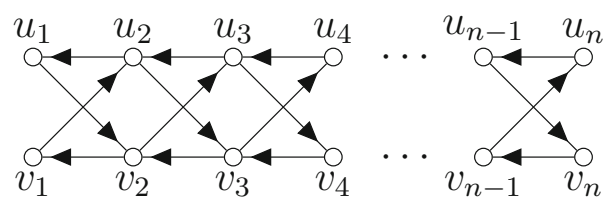

Let $D$ be an orientation of $G$. We call a pair of vertices $u$ and $v$ of $D$ full if $\theta_{D}(u, v)=\min \left\{\operatorname{deg}_{G}(u), \operatorname{deg}_{G}(v)\right\}$. We say that $D$ is saturated if every pair of distinct vertices is full, i.e., if $K(D)=P(G)$.

We now present some preliminary results. We begin with some straightforward bounds on $\bar{\kappa}_{\max }(G)$. From Robbins' theorem (Robbins 1939), we know that if $G$ is a 2-edge-connected graph, then $G$ has a strong orientation. This gives the following.

Theorem 2.2 If $G$ is a 2-edge-connected graph, then $\bar{\kappa}_{\max }(G) \geq 1$.

We remark, however, that we do not know whether every optimal orientation of a 2-edge-connected graph is strong.

If $D$ is an orientation of a graph of order $n$, then $\theta(u, v) \leq n-1$ for all pairs $u, v$ of vertices of $D$. This gives the following bound, first noted by Henning and Oellermann.

Theorem 2.3 (Henning and Oellermann 2004) If $G$ is a graph of order $n$, then $\bar{\kappa}_{\max }(G) \leq \frac{n-1}{2}$.

This bound is achieved, for example, if $n$ is odd and $G \cong K_{n}$ (Henning and Oellermann 2004).

We now turn to bounds on the ratio $\bar{\kappa}_{\max }(G) / \bar{\kappa}(G)$.

Theorem 2.4 For every graph $G$, we have $\bar{\kappa}_{\max }(G) / \bar{\kappa}(G) \leq 1$, and this bound is asymptotically sharp.

Proof If $D$ is an orientation of $G$, then $\theta_{D}(u, v) \leq 2 \kappa_{G}(u, v)$ for all pairs $u, v$ of vertices of $G$. So $\bar{\kappa}_{\max }(G) / \bar{\kappa}(G) \leq 1$.

To see that this bound is asymptotically sharp, let $F_{2 n}$ be the lexicographic product $P_{n} \circ\left(2 K_{1}\right)$, i.e., the graph obtained from two disjoint paths $P: v_{1} v_{2} \ldots v_{n}$ and $Q: u_{1} u_{2} \ldots u_{n}$ by adding the edges $v_{i} u_{i+1}$ and $u_{i} v_{i+1}$ for $1 \leq i<n$ (see Fig. 1). The graph $F_{2 n}$ has $n-2$ pairs $u, v$ such that $\kappa(u, v)=4$, namely those pairs $u_{i}, v_{i}$ for $2 \leq i \leq n-1$, and $4(n-3)$ pairs $u, v$ such that $\kappa(u, v)=3$, namely those pairs of adjacent vertices of degree four. For all remaining pairs $u, v$ of $F_{2 n}$, we see that $\kappa_{F_{2 n}}(u, v)=2$. Thus $\lim _{n \rightarrow \infty} \bar{\kappa}\left(F_{2 n}\right)=2$.

We now describe an orientation $D_{2 n}$ of $F_{2 n}$ with the property that $\lim _{n \rightarrow \infty} \frac{\bar{\kappa}\left(D_{2 n}\right)}{\bar{\kappa}\left(F_{2 n}\right)}=$ 1 (see Fig. 1). Orient $P$ from $v_{n}$ to $v_{1}$ and $Q$ from $u_{n}$ to $u_{1}$. The edges $u_{i} v_{i+1}$ and $v_{i} u_{i+1}$ are oriented as $\left(u_{i}, v_{i+1}\right)$ and $\left(v_{i}, u_{i+1}\right)$ for $1 \leq i<n$. For all pairs $u, v$ of vertices of degree 4 , we have $\theta_{D_{2 n}}(u, v)=4$. Since asymptotically almost all pairs of vertices have degree 4 , our claim follows. Hence, we have $\lim _{n \rightarrow \infty} \frac{\bar{\kappa}_{\max }\left(F_{2 n}\right)}{\bar{\kappa}\left(F_{2 n}\right)}=1$.

While Theorem 2.4 gives an asymptotically sharp upper bound on the ratio $\bar{\kappa}_{\max }(G) / \bar{\kappa}(G)$ for every graph $G$, it is an open problem to determine whether there is a positive constant $c$ such that for every connected graph $G$, we have $\bar{\kappa}_{\max }(G) / \bar{\kappa}(G) \geq c$. 
We briefly consider the edge connectivity analogue of $\bar{\kappa}_{\max }(G) / \bar{\kappa}(G)$ for the sake of comparison. Let $\lambda(u, v), \bar{\lambda}(G)$, and $\bar{\lambda}_{\max }(G)$ denote the edge connectivity analogues of $\kappa(u, v), \bar{\kappa}(G)$, and $\bar{\kappa}_{\max }(G)$, respectively. While the ratio $\bar{\kappa}_{\max }(G) / \bar{\kappa}(G)$ can be arbitrarily close to 1 , this is not the case for $\bar{\lambda}_{\max }(G) / \bar{\lambda}(G)$.

Theorem 2.5 For every graph $G$, we have $\bar{\lambda}_{\max }(G) / \bar{\lambda}(G) \leq 1 / 2$.

Proof Let $G$ be a graph with distinct vertices $u$ and $v$. Let $D$ be any orientation of $G$. There is a set of $\lambda_{G}(u, v)$ edges whose removal leaves a component $C_{u}$ containing $u$, and a component $C_{v}$ containing $v$. Suppose that exactly $k$ of the edges are oriented from a vertex of $C_{u}$ to a vertex of $C_{v}$. Every directed $u-v$ path in $D$ must use at least one of these edges, so $\lambda_{D}(u, v) \leq k$. Similarly, $\lambda_{D}(v, u) \leq \lambda_{G}(u, v)-k$. Thus, we have $\lambda_{D}(u, v)+\lambda_{D}(v, u) \leq \lambda_{G}(u, v)$ for every pair of distinct vertices $u, v$ of $G$, and the theorem statement follows immediately.

In proving that every $2 k$-edge-connected graph has a strongly $k$-edge-connected orientation, Nash-Williams actually proved the stronger result that for every graph $G$, there is an orientation $D$ of $G$ such that for every ordered pair $(u, v)$ of distinct vertices of $D$, there exist at least $\left\lfloor\lambda_{G}(u, v) / 2\right\rfloor$ edge-disjoint directed $u-v$ paths in $D$ (Nash-Williams 1960). It follows immediately that $\bar{\lambda}_{\max }(G) \geq \bar{\lambda}(D) \geq \frac{\bar{\lambda}(G)-1}{2}$, or equivalently,

$$
\frac{\bar{\lambda}_{\max }(G)}{\bar{\lambda}(G)} \geq \frac{1}{2}-\frac{1}{2 \bar{\lambda}(G)}
$$

We can easily obtain a positive constant lower bound on $\bar{\lambda}_{\max }(G) / \bar{\lambda}(G)$ in the case that $G$ is 2-edge-connected, and we will see that this bound is asymptotically sharp in Sect. 3.2.

Theorem 2.6 If $G$ is a 2-edge-connected graph, then $\bar{\lambda}_{\max }(G) / \bar{\lambda}(G) \geq 1 / 3$.

Proof Let $G$ be a 2-edge-connected graph. By the result of Nash-Williams, there is an orientation $D$ of $G$ such that for every pair of distinct vertices $u, v$ of $G$, we have $\lambda_{D}(u, v) \geq\left\lfloor\lambda_{G}(u, v) / 2\right\rfloor$. We claim that $\lambda_{D}(u, v)+\lambda_{D}(v, u) \geq \frac{2}{3} \lambda_{G}(u, v)$ for every pair of distinct vertices $u, v$ of $G$, from which the theorem statement easily follows. If $\lambda_{G}(u, v)$ is even, then we have

$$
\lambda_{D}(u, v)+\lambda_{D}(v, u) \geq 2 \cdot\left\lfloor\lambda_{G}(u, v) / 2\right\rfloor=\lambda_{G}(u, v) .
$$

On the other hand, if $\lambda_{G}(u, v)$ is odd, then since $G$ is 2-edge-connected, we have $\lambda_{G}(u, v) \geq 3$, and hence

$$
\lambda_{D}(u, v)+\lambda_{D}(v, u) \geq 2 \cdot\left\lfloor\lambda_{G}(u, v) / 2\right\rfloor=\lambda_{G}(u, v)-1 \geq \frac{2}{3} \lambda_{G}(u, v) .
$$

This completes the proof of the claim, and hence the theorem.

Overall, we see that the parameters $\bar{\lambda}_{\max }(G) / \bar{\lambda}(G)$ and $\bar{\kappa}_{\max }(G) / \bar{\kappa}(G)$ appear to behave rather differently in general. 


\section{Odd regular graphs}

In this section, we study bounds on the average connectivity of optimal orientations of $r$-regular $r$-connected graphs for odd $r$. A graph $G$ is uniformly $r$-connected if $\kappa(G)=\bar{\kappa}(G)=r$ [see Beineke et al. (2002)]. Clearly all $r$-regular $r$-connected graphs are uniformly $r$-connected.

Orientations of odd regular graphs are never saturated, due to the following elementary result.

Observation 3.1 Let $D$ be an orientation of an $r$-regular graph. If a pair of vertices $u, v$ in $D$ is full, then the out-degree of u equals the in-degree of $v$.

So, if $G \neq K_{2}$ is $r$-regular with saturated orientation $D$, then $r$ is even and the orientation of $D$ is regular; that is, every vertex has in- and out-degree $r / 2$. For even $r$, there do exist $r$-regular $r$-connected graphs with saturated orientations [see Henning and Oellermann (2001, 2004)].

\subsection{An upper bound on $\bar{\kappa}_{\max }(G)$}

Theorem 3.2 Let $r \geq 3$ be odd. If $G$ is an $r$-regular graph on $n$ vertices, then

$$
\bar{\kappa}_{\max }(G) \leq \frac{r-1}{2}+\frac{n}{4(n-1)} .
$$

Proof Let $D$ be an arbitrary orientation of $G$. Let $C$ be the number of full pairs in the orientation $D$. Then the total connectivity of $D$ is at most $C+(r-1) n(n-1) / 2$. Let $A$ be the vertices of $G$ with larger in-degree than out-degree. Then it follows from Observation 3.1 that a full pair contains one vertex belonging to $A$ and one vertex not belonging to $A$. So the number of full pairs of $D$ is at most $n^{2} / 4$. Our result now follows.

We now describe, for every odd $r \geq 3$, a family of $r$-regular graphs achieving the upper bound of Theorem 3.2. Let $s, t \geq 2$ be integers, and let $H_{s, t}$ be the graph constructed as follows. Take $2 s$ sets of vertices $V_{1}, \ldots, V_{2 s}$, each of size $t$. For every odd $i$, join every vertex in $V_{i}$ to every vertex in $V_{i+1}$. For every even $i$, add all edges between the vertices of $V_{i}$ and $V_{i+1}$ apart from a perfect matching (where subscripts are expressed modulo $2 s$ ). Then $H_{s, t}$ is a regular graph of degree $2 t-1$. For example, $H_{2,2}$ is the hypercube $Q_{3}$, and $H_{5,3}$ is shown in Fig. 2 .

Now, let $D_{s, t}$ be the orientation of $H_{s, t}$ obtained by placing the sets $V_{1}, V_{2}, \ldots, V_{2 s}$ around a circle and orienting edges clockwise (see Fig. 2). To aid us in our discussions, we colour the vertices in the odd subscripted sets white, and those in the even subscripted sets black. In $D_{s, t}$, the white vertices have in-degree $t-1$ and out-degree $t$, while the black vertices have out-degree $t-1$ and in-degree $t$. We claim that there are $t$ internally disjoint paths from any white vertex to any black vertex; and there are $t-1$ internally disjoint paths from any white vertex to any other white vertex, as well as from any black vertex to any other black vertex, and from any black vertex to any 
Fig. 2 The graph $H_{5,3}$

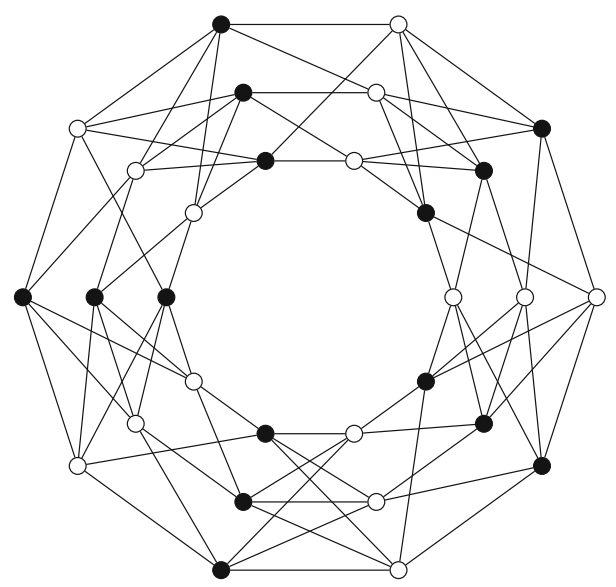

Fig. 3 The Möbius ladder of order 14

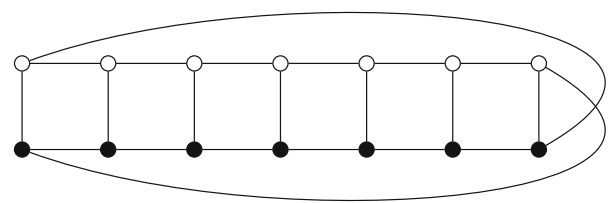

white vertex. It follows immediately that $\bar{\kappa}\left(D_{s, t}\right)=\bar{\kappa}_{\max }\left(H_{s, t}\right)$ matches the upper bound given by Theorem 3.2.

In the cubic case, the bound of Theorem 3.2 is also achieved by the Möbius ladders. Figure 3 shows a Möbius ladder of order 14.

\subsection{The average connectivity in 3-connected cubic graphs}

By Theorem 1.2, we have $\bar{\kappa}_{\max }(G) \geq 2$ for every 4-connected 4-regular graph $G$. In contrast, for a 3-connected cubic graph $G$, we show that $\bar{\kappa}_{\max }(G)$ can be arbitrarily close to 1 . This demonstrates that the lower bound of Theorem 2.2 is asymptotically sharp even for 3-connected cubic graphs. Additionally, we see that the ratio $\bar{\kappa}_{\max }(G) / \bar{\kappa}(G)$ can be arbitrarily close to $1 / 3$ for a 3 -connected cubic graph $G$ (and it cannot be smaller than $1 / 3$ ).

We begin by considering triangles in cubic graphs. Let $T$ be a triangle in a cubic graph $G$. In an orientation of the graph $G$ we define a vertex $u$ of $T$ as $b a d$ if $\theta(u, v) \leq 2$ for all vertices $v \in V(G)-V(T)$.

Lemma 3.3 Let $G$ be a cubic graph and let $T$ be a triangle in $G$. Then in any orientation of $G$, at least one vertex of $T$ is bad.

Proof Let $D$ be an orientation of $G$. Consider the three arcs incident with vertices of $T$, but not belonging to $T$. Suppose that $s$ of them are oriented away from $T$. In order to be in a full pair with a vertex not in $T$, a vertex of $T$ must have out-degree $s$. This is not possible for all vertices of $T$. 


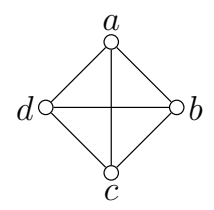

(a) The graph $K_{4}$

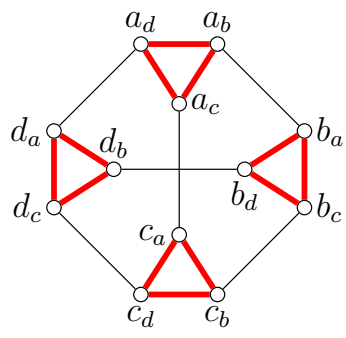

(b) The inflation $I\left(K_{4}\right)$

Fig. 4 The graphs $K_{4}$ and $I\left(K_{4}\right)$

Now, given a graph $G$, the graph $I(G)$, which we will call the inflation of $G$ [see Chvátal (1973)], is defined as the line graph of the subdivision of $G$ (where the subdivision of $G$ is obtained from $G$ by subdividing every edge exactly once). In the case that $G$ is a cubic graph, the process of constructing $I(G)$ is sometimes referred to as making the wye-delta replacement at each vertex of $G$. For a cubic graph $G$, the graph $I(G)$ is obtained as follows. For every vertex $v \in V(G)$, let $T_{v}$ be a triangle with vertices $v_{x}, v_{y}$, and $v_{z}$, where $x, y$, and $z$ are the neighbours of $v$ in $G$. The inflation $I(G)$ is obtained from the disjoint union $\cup_{v \in V(G)} T_{v}$ by joining $v_{x}$ with $x_{v}$, for every edge $v x$ of $G$. The inflation of $K_{4}$ is shown in Fig. 4.

If $F$ is an orientation of $I(G)$, then the orientation of $G$ obtained by assigning $v x$ the orientation $(v, x)$ if $\left(v_{x}, x_{v}\right)$ is in $I(G)$, or $(x, v)$ if $\left(x_{v}, v_{x}\right)$ is in $I(G)$, is called the projection of $F$ onto $G$. On the other hand, if $D$ is an orientation of $G$, and $F$ is an orientation of $I(G)$ with the property that $(v, x) \in E(D)$ implies $\left(v_{x}, x_{v}\right) \in E(F)$, then we say that $F$ is a lifting of $D$ to $I(G)$.

Lemma 3.4 Let $G$ be a cubic graph. Let $F$ be an orientation of $I(G)$ and let $D$ be the projection of $F$ onto $G$. If $D$ has exactly $C$ full pairs, then $F$ has at most $4 C+2|V(G)|$ full pairs.

Proof Suppose $T_{x}$ and $T_{y}$ are distinct triangles of $I(G)$. Then, by Lemma 3.3, there are at most four full pairs of vertices of $I(G)$ that have one vertex in $T_{x}$ and the other in $T_{y}$. If $P$ and $Q$ are (internally) disjoint $T_{x}-T_{y}$ paths in $I(G)$, then, apart from vertices in $T_{x}$ or $T_{y}$, the paths $P$ and $Q$ cannot contain vertices from the same triangle of $I(G)$. So $D$ has a pair of internally disjoint $x-y$ paths obtained from $P$ and $Q$ by contracting every triangle in $I(G)$ to the corresponding vertex in $G$. It follows that $x$ and $y$ must be a full pair in $D$. Thus, the number of full pairs of $F$ that are not contained in a single triangle is at most four times the number of full pairs of $D$.

Now it suffices to show that every triangle of $I(G)$ contains at most two full pairs of vertices. Suppose that $a$ and $b$ make up a full pair and belong to the same triangle $T_{z}$ of $I(G)$. If $\mathcal{F}_{a \rightarrow b}$ is a family of $\kappa_{I(G)}(a, b)$ internally disjoint $a-b$ paths, and $\mathcal{F}_{b \rightarrow a}$ is a family of $\kappa_{I(G)}(b, a)$ internally disjoint $b-a$ paths, then $\kappa_{I(G)}(a, b)+\kappa_{I(G)}(b, a)=3$, and every arc incident with $a$ and every arc incident with $b$ appears in $\mathcal{F}_{a \rightarrow b} \cup \mathcal{F}_{b \rightarrow a}$. So the arc between $a$ and $b$ constitutes one of the paths in this union, and a second path necessarily has length 2 and passes through the third vertex of $T_{z}$. The internal 
vertices of the third path in this union are necessarily not in $T_{z}$. Moreover, if the arc incident with $a$ but not in $T_{z}$ is directed away from $a$, then the arc incident with $b$ and not in $T_{z}$ is directed towards $b$, and vice versa. This can only happen for two pairs of vertices in $T_{z}$.

The previous lemma shows how orientations of $I(G)$ give rise to orientations of $G$ where connectedness properties between triangles in $I(G)$ translate to connectedness properties between the corresponding vertices of $G$. On the other hand, if $D$ is an orientation of $G$, and $F$ is a lifting of $D$ to $I(G)$ in which every triangle is oriented cyclically, then the next lemma establishes a connection between the connectedness properties of $D$ and those of $F$.

Lemma 3.5 Let $G$ be a 3-connected cubic graph, and let $D$ be a strong orientation of $G$. Let $F$ be a lifting of $D$ to $I(G)$ in which every triangle is oriented cyclically. If $D$ has exactly $C$ full pairs, then $F$ has $4 C+2|V(G)|$ full pairs.

Proof Note that the orientation $F$ of $I(G)$ is strong. Consider a full pair $x$ and $y$ in $D$. Without loss of generality, assume that $x$ has out-degree 2 and $y$ has in-degree 2 . Let $a_{1}, a_{2}$ be the out-neighbours and $a_{3}$ the in-neighbour of $x$ in $D$. Let $b_{1}, b_{2}$ be the in-neighbours and $b_{3}$ the out-neighbour of $y$ in $D$. Then for $i \in\{1,2\}$ and $j \in\{1,2\}$, there are two disjoint paths from $x_{a_{i}}$ to $y_{b_{j}}$ in $I(G)$. It follows that for two distinct triangles in $I(G)$, we have four full pairs of vertices in $I(G)$ (where each pair contains a vertex from each of these two triangles).

Since every arc in $D$ is in a cycle, it follows that for $i \in\{1,2\}$ there is a path from $x_{a_{i}}$ to $x_{a_{3}}$ that is internally disjoint from $T_{x}$. Thus for every triangle $T_{x}$ in $D$, there are two full pairs with both vertices in $T_{x}$. This gives the desired result.

Together, Lemmas 3.4 and 3.5 give the following.

Theorem 3.6 Let $G$ be a 3-connected cubic graph of order n. If G has a strong optimal orientation $D$, and $F$ is a lifting of $D$ to $I(G)$ in which every triangle is oriented cyclically, then $F$ is a strong optimal orientation of $I(G)$. Moreover,

$$
\bar{\kappa}_{\max }(I(G))=\bar{\kappa}(F)=1+\frac{4 n(n-1)\left[\bar{\kappa}_{\max }(G)-1\right]+2 n}{3 n(3 n-1)} .
$$

Proof Suppose $G$ has a strong optimal orientation $D$. Then $\theta_{D}(u, v) \geq 2$ for all pairs $u, v$ of vertices of $D$. Also if $u, v$ is a full pair, then $\theta_{D}(u, v)-2=1$. Hence

$$
\sum_{\{u, v\} \subseteq V(G)}(\theta(u, v)-2)=K(D)-n(n-1)
$$

equals the number of full pairs in $D$. If $F$ is the orientation of $I(G)$ obtained by lifting $D$ to $I(G)$ and orienting each triangle cyclically, then, by Lemma 3.5, $F$ has $4(K(D)-n(n-1))+2 n$ full pairs. Thus

$$
K(F)=3 n(3 n-1)+4(K(D)-n(n-1))+2 n=3 n(3 n-1)+4 n(n-1)[\bar{\kappa}(D)-1]+2 n .
$$


The result now follows.

It is readily seen that $K_{4}$ has a strong optimal orientation, and that $\bar{\kappa}_{\max }\left(K_{4}\right)=4 / 3$. Thus we obtain the following corollary to Theorem 3.6.

Corollary $3.7 \lim _{k \rightarrow \infty} \bar{\kappa}_{\max }\left(I^{k}\left(K_{4}\right)\right)=1$.

We conclude that the bound of Theorem 2.2 is asymptotically sharp even for 3connected cubic graphs, and that the ratio $\bar{\kappa}_{\max }(G) / \bar{\kappa}(G)$ can be made arbitrarily close to $1 / 3$ for a 3 -connected cubic graph $G$.

Finally, we note that for every pair of distinct vertices $u, v$ in a cubic graph, a collection of $u-v$ paths is internally disjoint if and only if it is edge disjoint. Hence, if $G$ is a cubic graph, then $\bar{\lambda}_{\text {max }}(G) / \bar{\lambda}(G)=\bar{\kappa}_{\max }(G) / \bar{\kappa}(G)$. Therefore, it follows from Corollary 3.7 that the bound of Theorem 2.6 is asymptotically sharp.

\section{Orientations of minimally 2-connected graphs}

It is natural to ask which graphs $G$ satisfy $\kappa(G)=\bar{\kappa}(G)=k$ for some positive integer $k$. It was observed in Beineke et al. (2002) that every graph $G$ having this property is minimally $k$-connected, i.e., satisfies $\kappa(G)=k$ and $\kappa(G-e)<k$ for every edge $e$ of $G$. The minimally 1 -connected graphs are precisely the trees, whose average connectivity is 1 . However, the average connectivity of minimally $k$-connected graphs, for $k \geq 2$, need not be $k$. Indeed, it has been shown that if $G$ is a minimally 2-connected graph, then $2 \leq \bar{\kappa}(G)<9 / 4$, and these bounds are asymptotically tight (Casablanca et al. $2021)$. In this section, we determine bounds on $\bar{\kappa}_{\max }(G)$ and $\bar{\kappa}_{\max }(G) / \bar{\kappa}(G)$ for every minimally 2-connected graph $G$.

Mader (1972) showed that if $G$ is minimally $k$-connected, then the subgraph of $G$ induced by the vertices of degree exceeding $k$ is a forest. For minimally 2 -connected graphs, the following stronger result holds.

Theorem 4.1 (see Bollobás (2004, Theorem 3.8)) Let $G$ be a minimally 2-connected graph that is not a cycle, and let $F$ be the subgraph induced by the vertices of degree exceeding 2 in $G$. Then $F$ is a forest with at least two components.

Minimally 2-connected graphs were characterized independently by Dirac (1967) and Plummer (1968). A cycle $C$ of a graph $G$ is said to have a chord if there is an edge of $G$ that joins a pair of non-adjacent vertices from $C$. Plummer characterized the minimally 2-connected graphs as follows.

Theorem 4.2 (Plummer 1968) A 2-connected graph $G$ is minimally 2-connected if and only if no cycle of $G$ has a chord.

\subsection{Bounds on $\bar{\kappa}_{\max }(G)$}

Here, we establish upper and lower bounds on $\bar{\kappa}_{\max }(G)$, where $G$ is a minimally 2connected graph. Our first lemma concerns optimal orientations of connected graphs in general. 
Lemma 4.3 Let D be an optimal orientation of a connected graph $G$ of order at least 3. Then no arc of $D$ is oriented from a source to a sink.

Proof Suppose otherwise that $D$ contains the arc $(u, v)$, where $u$ is a source and $v$ is a sink. Then the only oriented path in $D$ that contains the $\operatorname{arc}(u, v)$ is the path of length 1 from $u$ to $v$. Let $D^{\prime}$ be the orientation obtained by reversing the arc $(u, v)$ to obtain $(v, u)$. Note that $\theta_{D^{\prime}}(u, v)=\theta_{D}(u, v)$, and that $\theta_{D^{\prime}}(x, y) \geq \theta_{D}(x, y)$ for any other pair of vertices in $G$, since no path between $x$ and $y$ used the arc $(u, v)$. Further, there is a vertex $w \neq v$ such that either $(u, w)$ or $(w, v)$ is an $\operatorname{arc}$ in $D$, and there is an oriented path from $v$ to $w$ (or $w$ to $u$, respectively) in $D^{\prime}$, while there was none in $D$. This gives $\bar{\kappa}\left(D^{\prime}\right)>\bar{\kappa}(D)$, a contradiction.

We now establish a structure result for every minimally 2-connected graph $G$ of a given order for which $\bar{\kappa}_{\max }(G)$ has largest possible value.

Lemma 4.4 Let $G$ be a minimally 2-connected graph of order $n \geq 5$ such that $\bar{\kappa}_{\max }(G)$ is largest. Then no two vertices of degree 2 are adjacent in $G$.

Proof Let $G$ be as in the lemma statement. Since $K_{2, n-2}$ has an orientation for which the total connectivity exceeds $n(n-1)$, we know that $G$ is not a cycle. Suppose, towards a contradiction, that $G$ has two adjacent vertices of degree 2, say $u$ and $v$. Let $u^{\prime}$ be the other neighbour of $u$ and let $v^{\prime}$ be the other neighbour of $v$. Since $G$ is 2-connected and $n \geq 5$, we have $u^{\prime} \neq v^{\prime}$. Further, since $G$ is minimally 2-connected and is not a cycle, one can argue that $u^{\prime} v^{\prime} \notin E(G)$ by using Theorem 4.2. Let $G^{\prime}$ be the graph obtained by deleting the edge $u v$ and adding the edges $u^{\prime} v$ and $u v^{\prime}$. Using the fact that $u^{\prime} v^{\prime} \notin E(G)$, it is straightforward to show that $G^{\prime}$ is minimally 2-connected. We claim that $\bar{\kappa}_{\text {max }}\left(G^{\prime}\right)>\bar{\kappa}_{\text {max }}(G)$.

Let $D$ be an optimal orientation of $G$. Suppose first that the path $P: u^{\prime} u v v^{\prime}$ is oriented from $u^{\prime}$ to $v^{\prime}$ or from $v^{\prime}$ to $u^{\prime}$ in $D$. Let $D^{\prime}$ be the orientation of $G^{\prime}$ obtained from $D$ by deleting the arcs incident to $u$ and $v$, and adding the $\operatorname{arcs}\left(u^{\prime}, u\right),\left(u, v^{\prime}\right),\left(v^{\prime}, v\right)$, and $\left(v, u^{\prime}\right)$. By straightforward arguments, we have $\theta_{D^{\prime}}\left(u^{\prime}, v^{\prime}\right)=\theta_{D}\left(u^{\prime}, v^{\prime}\right)+1$, and $\theta_{D^{\prime}}(x, y) \geq \theta_{D}(x, y)$ for every other unordered pair of vertices $x, y$. It follows that $\bar{\kappa}\left(D^{\prime}\right)>\bar{\kappa}(D)$.

On the other hand, if the vertices of $P$ do not induce an oriented path in $D$, then either $u$ or $v$ is a source or a sink in $D$. Suppose $u$ is a sink; the other cases are similar. Then by Lemma 4.3, $v$ is not a source. So $\left(u^{\prime}, u\right),(v, u)$, and $\left(v^{\prime}, v\right)$ are $\operatorname{arcs}$ in $D$. Let $D^{\prime}$ be the orientation of $G^{\prime}$ obtained from $D$ by deleting the arc $(v, u)$ and adding the $\operatorname{arcs}\left(v^{\prime}, u\right)$ and $\left(v, u^{\prime}\right)$. Once again, one can verify that $\theta_{D^{\prime}}\left(u^{\prime}, v^{\prime}\right)=\theta_{D}\left(u^{\prime}, v^{\prime}\right)+1$, and that $\theta_{D^{\prime}}(x, y) \geq \theta_{D}(x, y)$ for every other unordered pair of vertices $x, y$. It follows that $\bar{\kappa}\left(D^{\prime}\right)>\bar{\kappa}(D)$.

We are now ready to bound the value of $\bar{\kappa}_{\max }(G)$ for any minimally 2 -connected graph $G$.

Theorem 4.5 Let $G$ be a minimally 2-connected graph of order $n \geq 3$. Then

$$
1 \leq \bar{\kappa}_{\max }(G) \leq 1+\frac{(n-3)^{2}}{4 n(n-1)}<\frac{5}{4}
$$


Proof The lower bound follows immediately from Robbins' Theorem. For the upper bound, let $G$ be a minimally 2 -connected graph of order $n \geq 3$ such that $\bar{\kappa}_{\text {max }}(G)$ is largest. Suppose that $G$ has $s$ vertices of degree at least 3 and $n-s$ vertices of degree 2. Let $V_{1}=\left\{v_{1}, \ldots, v_{s}\right\}$ be the set of vertices of degree at least 3 . Let $d_{i}=\operatorname{deg}\left(v_{i}\right)$ for all $i \in\{1, \ldots, s\}$. By Theorem 4.1, the subgraph $G\left[V_{1}\right]$ is a forest with at least two components. So the subgraph induced by the vertices of degree at least 3 has at most $s-2$ edges. By Lemma 4.4, every edge incident to a vertex of degree 2 must also be incident to a vertex of degree at least 3 . Thus we have

$$
\sum_{i=1}^{s} d_{i}=2(n-s)+2\left|E\left(G\left[V_{1}\right]\right)\right| \leq 2(n-s)+2(s-2)=2 n-4
$$

Let $D$ be some orientation of $G$, and let $d$ and $r$ be the unique integers such that $0 \leq r<s$ and $2 n-4=d s+r$. By Observation 2.1, we have

$$
\begin{aligned}
K(D) \leq P(G) & =2 \cdot\left[\left(\begin{array}{c}
n \\
2
\end{array}\right)-\left(\begin{array}{c}
s \\
2
\end{array}\right)\right]+P\left(d_{1}, d_{2}, \ldots, d_{s}\right) \\
& \leq 2 \cdot\left[\left(\begin{array}{c}
n \\
2
\end{array}\right)-\left(\begin{array}{c}
s \\
2
\end{array}\right)\right]+d\left(\begin{array}{c}
s \\
2
\end{array}\right)+\left(\begin{array}{c}
r \\
2
\end{array}\right) \\
& =2\left(\begin{array}{l}
n \\
2
\end{array}\right)+(d-2)\left(\begin{array}{l}
s \\
2
\end{array}\right)+\left(\begin{array}{c}
r \\
2
\end{array}\right) \\
& =n(n-1)+\left[\frac{2 n-4-r}{s}-2\right] \cdot\left(\begin{array}{c}
s \\
2
\end{array}\right)+\left(\begin{array}{c}
r \\
2
\end{array}\right) \\
& =n(n-1)+\left[\frac{2 n-2 s-4-r}{s}\right]\left(\begin{array}{l}
s \\
2
\end{array}\right)+\left(\begin{array}{c}
r \\
2
\end{array}\right) \\
& =n(n-1)+(n-s-2)(s-1)-\frac{r(s-1)}{2}+\frac{r(r-1)}{2} \\
& =n(n-1)+(n-s-2)(s-1)-\frac{r(s-r)}{2} \\
& \leq n(n-1)+(n-s-2)(s-1) .
\end{aligned}
$$

By elementary calculus, this last expression is at most $n(n-1)+\frac{(n-3)^{2}}{4}$, with equality if and only if $s=\frac{n-1}{2}$. Since $D$ is an arbitrary orientation of $G$, it follows that $\bar{\kappa}_{\max }(G) \leq 1+\frac{(n-3)^{2}}{4 n(n-1)}<\frac{5}{4}$.

The lower bound of Theorem 4.5 is sharp if and only if $G$ is a cycle. We believe that the upper bound of Theorem 4.5 can be improved. We will see later in Example 4.10 that $\bar{\kappa}_{\max }(G)$ can be made arbitrarily close to $9 / 8$ for a minimally 2 -connected graph $G$; we know of several distinct families of minimally 2-connected graphs which demonstrate this, but we do not know of any minimally 2 -connected graph $G$ with $\bar{\kappa}_{\max }(G)>9 / 8$.

\subsection{Bounds on the ratio $\bar{\kappa}_{\max }(G) / \bar{K}(G)$}

The following bounds follow from Theorem 4.5 and from a bound on the average connectivity of a minimally 2-connected graph given in Casablanca et al. (2021).

Corollary 4.6 Let $G$ be a minimally 2-connected graph. Then

$$
\frac{4}{9}<\frac{\bar{\kappa}_{\max }(G)}{\bar{\kappa}(G)}<\frac{5}{8}
$$


Proof The lower bound follows from the facts that $\bar{\kappa}_{\max }(G) \geq 1$ (by Theorem 2.2), and $\bar{\kappa}(G)<\frac{9}{4}$ [by Casablanca et al. (2021, Theorem 2.11)]. The upper bound follows from the facts that $\bar{\kappa}_{\max }(G)<\frac{5}{4}$ (by Theorem 4.5), and $\bar{\kappa}(G) \geq 2$ since $G$ is 2-connected.

We do not know whether these bounds are sharp. In the remainder of this section, we describe constructions of minimally 2-connected graphs $G$ with ratio $\bar{\kappa}_{\max }(G) / \bar{\kappa}(G)$ arbitrarily close to $\frac{25}{54}$ (which is $\frac{1}{54}$ greater than the lower bound), and minimally 2connected graphs with ratio $\bar{\kappa}_{\max }(G) / \bar{\kappa}(G)$ arbitrarily close to $\frac{9}{16}$ (which is $\frac{1}{16}$ less than the upper bound).

We begin with two short lemmas on subdivisions of graphs. For a graph $G$, we let $S(G)$ denote the subdivision of $G$, obtained from $G$ by subdividing every edge exactly once. For ease of notation, we let $S(G)$ have vertex set $V(G) \cup E(G)$, and $u \in V(G)$ and $e \in E(G)$ are joined by an edge in $S(G)$ whenever $u$ is an endvertex of $e$ in $G$. Note that if $G$ is 2-connected, then $S(G)$ is minimally 2-connected.

Lemma 4.7 For any graph $G$ of order $n$ and size $m$,

$$
K(S(G)) \leq 2\left[\left(\begin{array}{c}
n+m \\
2
\end{array}\right)-\left(\begin{array}{c}
n \\
2
\end{array}\right)\right]+K(G),
$$

with equality if and only if $G$ is 2-connected.

Proof Let $u$ and $v$ be distinct vertices of $S(G)$. If either $u$ or $v$ is in $E(G)$, then $\kappa_{S(G)}(u, v) \leq 2$, with equality for all such pairs if and only if $G$ is 2-connected. Suppose otherwise that $u, v \in V(G)$. Any collection of $k$ internally disjoint $u-v$ paths in $S(G)$ corresponds to a collection of $k$ internally disjoint $u-v$ paths in $G$ in an obvious manner; so $\kappa_{S(G)}(u, v)=\kappa_{G}(u, v)$. The statement now follows by summing the connectivities between all pairs of vertices.

Lemma 4.8 Let $G$ be a graph of order $n$ and size $m$. Then

$$
K_{\max }(S(G)) \leq 2\left[\left(\begin{array}{c}
n+m \\
2
\end{array}\right)-\left(\begin{array}{c}
n \\
2
\end{array}\right)\right]+K_{\max }(G),
$$

with equality if there is an optimal orientation of $G$ that is strong.

Proof Let $D_{S}$ be any orientation of $S(G)$. Let $u$ and $v$ be distinct vertices of $S(G)$. If either $u$ or $v$ is in $E(G)$, then $\theta_{D_{S}}(u, v) \leq 2$, with equality for all such pairs if $D_{S}$ is strong. Suppose otherwise that $u, v \in V(G)$. Consider the partial orientation $D$ of $G$ obtained from $D_{S}$ as follows. For every edge $e \in E(G)$, say $e=u v$, orient $e$ from $u$ to $v$ if and only if both of the arcs $(u, e)$ and $(e, v)$ appear in $D_{S}$. Remove all edges of $G$ that are not given an orientation in this manner (namely those edges of $G$ that are sources or sinks as vertices in $\left.D_{S}\right)$. Then $\kappa_{D_{S}}(u, v)=\kappa_{D}(u, v)$. Thus, we have

$$
\sum_{u, v \in V(G)} \kappa_{D_{S}}(u, v)=\sum_{u, v \in V(G)} \kappa_{D}(u, v) \leq K_{\max }(G),
$$


with equality if and only if $D$ is an optimal orientation of $G$ (in particular, every edge of $G$ must be in $D$ ). Altogether, we have

$$
K_{\max }(S(G)) \leq 2\left[\left(\begin{array}{c}
n+m \\
2
\end{array}\right)-\left(\begin{array}{c}
n \\
2
\end{array}\right)\right]+K_{\max }(G),
$$

with equality if $D$ is a strong optimal orientation of $G$.

Example 4.9 Let $G_{k}=I^{k}\left(K_{4}\right)$, with notation as in Sect. 3.2. The subdivision $S\left(G_{k}\right)$ of $G_{k}$ is minimally 2-connected (since $G_{k}$ is 2-connected), and we show that

$$
\lim _{k \rightarrow \infty} \frac{\bar{\kappa}_{\max }\left(S\left(G_{k}\right)\right)}{\bar{\kappa}\left(S\left(G_{k}\right)\right)}=\frac{25}{54}
$$

For ease of notation, let $n=4 \cdot 3^{k}$ (the order of $G_{k}$ ), let $m=\frac{3 n}{2}$ (the size of $G_{k}$ ), and let $N=\left(\begin{array}{c}n+m \\ 2\end{array}\right)-\left(\begin{array}{l}n \\ 2\end{array}\right)$. By a straightforward computation, we have

$$
\lim _{k \rightarrow \infty} \frac{2 N}{n(n-1)}=\frac{21}{4}
$$

By Theorem 3.6, we know that $G_{k}$ has a strong optimal orientation. Hence, by Lemmas 4.7 and 4.8 , we have

$$
\frac{\bar{\kappa}_{\max }\left(S\left(G_{k}\right)\right)}{\bar{\kappa}\left(S\left(G_{k}\right)\right)}=\frac{K_{\max }\left(S\left(G_{k}\right)\right)}{2 K\left(S\left(G_{k}\right)\right)}=\frac{2 N+K_{\max }\left(G_{k}\right)}{2\left[2 N+K\left(G_{k}\right)\right]}=\frac{\frac{2 N}{n(n-1)}+\bar{\kappa}_{\max }\left(G_{k}\right)}{\frac{4 N}{n(n-1)}+\bar{\kappa}\left(G_{k}\right)} .
$$

Now using (1), (2), Corollary 3.6, and the fact that $\bar{\kappa}\left(G_{k}\right)=3$, we obtain

$$
\lim _{k \rightarrow \infty} \frac{\bar{\kappa}_{\max }\left(S\left(G_{k}\right)\right)}{\bar{\kappa}\left(S\left(G_{k}\right)\right)}=\frac{\frac{21}{4}+1}{\frac{21}{2}+3}=\frac{25}{54} .
$$

Example 4.10 Let $n$ be a positive integer, and define $H_{4 n+1}$ as follows (see Fig. 5). Let $P: v_{1} v_{2} \ldots v_{2 n}$ be a path of order $2 n$. Subdivide each edge $v_{i} v_{i+1}$ of $P$ for $1 \leq i<2 n$, and call the new vertex $w_{i}$. Now add a vertex $w_{0}$ and join it to $v_{1}$ and $v_{2}$, and add a vertex $w_{2 n}$ and join it to $v_{2 n-1}$ and $v_{2 n}$. To complete the construction of $H_{4 n+1}$, add the edges $v_{i} v_{i+2}$ for $1 \leq i<2 n-1$. By inspection, $H_{4 n+1}$ is minimally 2-connected, and we now show that

$$
\lim _{n \rightarrow \infty} \frac{\bar{\kappa}_{\max }\left(H_{4 n+1}\right)}{\bar{\kappa}\left(H_{4 n+1}\right)} \geq \frac{9}{16}
$$

Apart from the pairs of vertices $v_{i}, v_{i+1}$ for $1 \leq i<2 n$, the connectivity between any pair of vertices $u, v$ in $H_{4 n+1}$ is 2 . So the total connectivity of $H_{4 n+1}$ is

$$
K\left(H_{4 n+1}\right)=2\left(\begin{array}{c}
4 n+1 \\
2
\end{array}\right)+2 n-1
$$




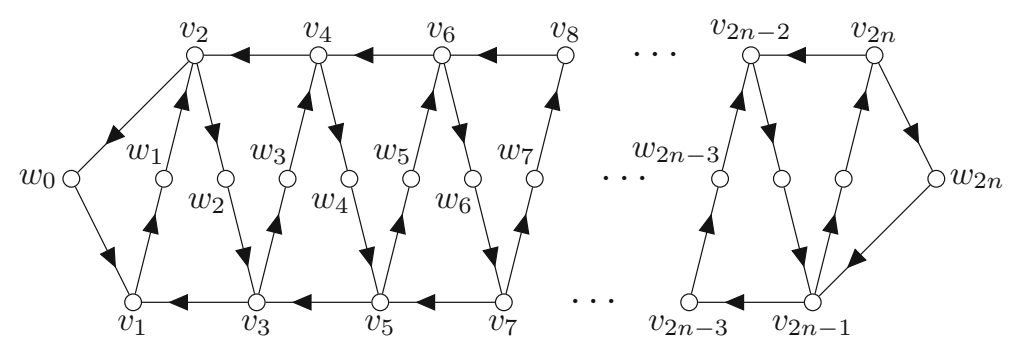

Fig. 5 A family of minimally 2-connected graphs with ratio tending to $\frac{9}{16}$

Hence, $\lim _{n \rightarrow \infty} \bar{\kappa}\left(H_{4 n+1}\right)=2$.

We now describe an orientation $D_{4 n+1}$ of $H_{4 n+1}$ (see Fig. 5). For every $1 \leq i<2 n$, orient the path $v_{i} w_{i} v_{i+1}$ from $v_{i}$ to $v_{i+1}$. Orient the path $v_{2} w_{0} v_{1}$ from $v_{2}$ to $v_{1}$, and orient the path $v_{2 n} w_{2 n} v_{2 n-1}$ from $v_{2 n}$ to $v_{2 n-1}$. Orient the path $v_{2 n} v_{2 n-2} \ldots v_{2}$ from $v_{2 n}$ to $v_{2}$, and the path $v_{2 n-1} v_{2 n-3} \ldots v_{1}$ from $v_{2 n-1}$ to $v_{1}$. One can verify that $D_{4 n+1}$ is strong. Note that $H_{4 n+1}$ has $2 n$ vertices of degree at least 3 (the $v_{i}$ 's) and $2 n+1$ vertices of degree 2 (the $w_{i}$ 's). Note that for any pair $v_{i}, v_{j}$ with $i<j$, we have $\kappa_{D_{4 n+1}}\left(v_{i}, v_{j}\right)=1$ and $\kappa_{D_{4 n+1}}\left(v_{j}, v_{i}\right)=2$, hence $\theta_{D_{4 n+1}}\left(v_{i}, v_{j}\right)=3$. Therefore, the total connectivity of $D_{4 n+3}$ is given by

$$
K\left(D_{4 n+1}\right)=2\left(\begin{array}{c}
4 n+1 \\
2
\end{array}\right)+\left(\begin{array}{c}
2 n \\
2
\end{array}\right) .
$$

So $\lim _{n \rightarrow \infty} \bar{\kappa}\left(D_{4 n+1}\right)=\frac{9}{8}$, and we certainly have $\bar{\kappa}_{\text {max }}\left(H_{4 n+1}\right) \geq \bar{\kappa}\left(D_{4 n+1}\right)$ for all $n$. It follows that

$$
\lim _{n \rightarrow \infty} \frac{\bar{\kappa}_{\max }\left(H_{4 n+1}\right)}{\bar{\kappa}\left(H_{4 n+1}\right)} \geq \lim _{n \rightarrow \infty} \frac{\bar{\kappa}\left(D_{4 n+1}\right)}{\bar{\kappa}\left(H_{4 n+1}\right)}=\frac{9}{16} .
$$

\section{Maximal outerplanar graphs and 2-trees}

A graph is outerplanar if it can be drawn in the plane so that no two of its edges cross and all of its vertices appear on the boundary of the outer face. A graph is maximal outerplanar if it is outerplanar and the addition of any edge destroys this property. It is known that every maximal outerplanar graph is a 2-tree. For $k \geq 1$, the $k$-trees are defined recursively as follows: the complete graph $K_{k}$ is a $k$-tree, and if $T$ is a $k$-tree, then the graph obtained from $T$ by adding a new vertex and joining it to every vertex in a $k$-clique of $T$ is a $k$-tree. Note that trees are precisely the 1 -trees.

It was shown in Dankelmann and Oellermann (2003) that all maximal outerplanar graphs of the same order have the same average connectivity. 
Theorem 5.1 (Dankelmann and Oellermann 2003) If $G$ is a maximal outerplanar graph of order $n$, then

$$
\bar{\kappa}(G)=2+\frac{2 n-6}{n(n-1)} .
$$

So, for maximal outerplanar graphs of a fixed order, the ratio $\bar{\kappa}_{\max }(G) / \bar{\kappa}(G)$ is maximized or minimized exactly when $\bar{\kappa}_{\max }(G)$ is maximized or minimized, respectively. So it suffices to focus on bounds for $\bar{\kappa}_{\max }(G)$ if $G$ is maximal outerplanar. We show that if $G$ is a maximal outerplanar graph, then $\bar{\kappa}_{\text {max }}(G) \leq \frac{3}{2}+o(1)$. Moreover, this bound is asymptotically sharp. We conjecture that $\bar{\kappa}_{\max }(G) \geq 19 / 18$ for every maximal outerplanar graph of order at least 4 , and we demonstrate that if this conjecture is true, then the bound is asymptotically sharp.

As was the case for maximal outerplanar graphs, one can show (by induction), that if $G$ is a 2-tree of order $n \geq 3$, then $\bar{\kappa}(G)=2+\frac{2 n-6}{n(n-1)}$. Thus, for 2-trees of a fixed order, the ratio $\bar{\kappa}_{\max }(G) / \bar{\kappa}(G)$ is maximized or minimized exactly when $\bar{\kappa}_{\max }(G)$ is maximized or minimized, respectively. We show that if $G$ is a 2 -tree, then the bound $\bar{\kappa}_{\max }(G) \geq 1$, guaranteed by Robbins' Theorem, is asymptotically sharp.

\subsection{Maximal outerplanar graphs}

We use the following notation throughout this section. Let $G$ be a maximal outerplanar graph with a given embedding in the plane. We say that an edge is an outer edge of $G$ if it is part of the cycle $C$ which forms the boundary of the outer face of $G$. An edge that is not an outer edge is a chord of $G$.

The weak dual $G^{*}$ of $G$ is the graph whose vertices are the faces of $G$ distinct from the outer face, and two vertices of $G^{*}$ are adjacent if, as faces of $G$, their boundaries share an edge. If $u^{*}$ is a vertex of $G^{*}$, then $V\left(u^{*}\right)$ denotes the set of vertices of $G$ that are on the boundary of $u^{*}$. It is well-known that $G^{*}$ is a tree of maximum degree at most 3 , and it is easy to see that a vertex of $G^{*}$ has degree 3 if and only if its boundary consists of three chords.

Lemma 5.2 Let $G$ be a maximal outerplanar graph of order $n$. Let $A$ be the set of vertices of $G$ that are on a 4-cycle whose edges are chords. Let $B_{2}$ be the set of vertices of degree 2 in $G$. Then

$$
\left|B_{2}\right| \geq \frac{1}{2}|A|+2
$$

Proof Denote the set of vertices of $G^{*}$ whose degree is 3 and who are adjacent to some other vertex of degree 3 by $V_{3}^{*}$. We prove the lemma by bounding $\left|V_{3}^{*}\right|$ from above in terms of $\left|B_{2}\right|$, and from below in terms of $|A|$.

We first bound $\left|V_{3}^{*}\right|$ from above in terms of $\left|B_{2}\right|$. Since a vertex of $G$ has degree 2 if and only if its incident edges are both on the outer cycle, there is a natural bijection between the vertices of $G$ of degree 2 and the leaves of $G^{*}$. Hence $\left|B_{2}\right|=n_{1}^{*}$, where $n_{i}^{*}$ is the number of vertices of $G^{*}$ of degree $i$. On the other hand, since $G^{*}$ is a tree 
of maximum degree at most 3 , we have $n_{3}^{*}=n_{1}^{*}-2$. Hence

$$
\left|V_{3}^{*}\right| \leq n_{3}^{*}=n_{1}^{*}-2=\left|B_{2}\right|-2
$$

We now bound $\left|V_{3}^{*}\right|$ from below in terms of $|A|$. We first show that

$$
A=\bigcup_{u^{*} \in V_{3}^{*}} V\left(u^{*}\right) .
$$

To see that $A \subseteq \bigcup_{u^{*} \in V_{3}^{*}} V\left(u^{*}\right)$, let $v \in A$. Then $v$ is on some 4-cycle $C_{v}$ whose edges are chords. Since $G$ is maximal outerplanar, $C_{v}$ also has a chord $e$. The two faces $u_{1}^{*}$ and $u_{2}^{*}$ of $G$ that have $e$ on their respective boundaries are adjacent in $G^{*}$. Further, since the edges on their boundaries are all chords, we see that $u_{1}^{*}$ and $u_{2}^{*}$ have degree 3 in $G^{*}$. Hence, we have $u_{1}^{*}, u_{2}^{*} \in V_{3}^{*}$ and $v \in V\left(u_{1}^{*}\right) \cup V\left(u_{2}^{*}\right)$, and it follows that $v \in \bigcup_{u^{*} \in V_{3}^{*}} V\left(u^{*}\right)$.

To see that $\bigcup_{u^{*} \in V_{3}^{*}} V\left(u^{*}\right) \subseteq A$, let $v \in \bigcup_{u^{*} \in V_{3}^{*}} V\left(u^{*}\right)$. Then $v \in V\left(u_{1}^{*}\right)$ for some $u_{1}^{*} \in V_{3}^{*}$. By the definition of $V_{3}^{*}$, the vertex $u_{1}^{*}$ has a neighbour $u_{2}^{*}$ in $G^{*}$ of degree 3. Since $V\left(u_{1}^{*}\right)$ and $V\left(u_{2}^{*}\right)$ share an edge of $G$, and since the edges on the boundary of $u_{i}^{*}$, for $i \in\{1,2\}$, are all chords, there is a 4-cycle of $G$ containing the vertices of $V\left(u_{1}^{*}\right) \cup V\left(u_{2}^{*}\right)$, whose edges are chords. Hence $v \in A$. This proves (4).

Let $H_{1}^{*}, H_{2}^{*}, \ldots, H_{k}^{*}$ be the components of the graph $G^{*}\left[V_{3}^{*}\right]$. Then each $H_{i}^{*}$ is a tree on at least two vertices. Clearly, if $H_{i}^{*}$ has two vertices, then $\left|\bigcup_{u^{*} \in V\left(H_{i}^{*}\right)} V\left(u^{*}\right)\right|=$ 4. Every additional vertex in $H_{i}^{*}$ increases $\left|\bigcup_{u^{*} \in V\left(H_{i}^{*}\right)} V\left(u^{*}\right)\right|$ by one, hence

$$
\left|\bigcup_{u^{*} \in V\left(H_{i}^{*}\right)} V\left(u^{*}\right)\right|=\left|V\left(H_{i}^{*}\right)\right|+2 \leq 2\left|V\left(H_{i}^{*}\right)\right|
$$

Summation over $i=1,2, \ldots, k$ yields

$$
\begin{aligned}
|A| & =\left|\bigcup_{u^{*} \in V_{3}^{*}} V\left(u^{*}\right)\right| \\
& =\left|\bigcup_{i=1}^{k} \bigcup_{u^{*} \in V\left(H_{i}\right)} V\left(u^{*}\right)\right| \\
& \leq \sum_{i=1}^{k}\left|\bigcup_{u^{*} \in V\left(H_{i}\right)} V\left(u^{*}\right)\right| \\
& \leq \sum_{i=1}^{k} 2\left|V\left(H_{i}^{*}\right)\right| \\
& =2\left|V_{3}^{*}\right| .
\end{aligned}
$$

Combining (3) with (5) now yields the statement of the lemma. 
Theorem 5.3 If $G$ is a maximal outerplanar graph of order $n$, then

$$
\bar{\kappa}_{\text {max }}(G) \leq \frac{3}{2}+\frac{n-5}{n(n-1)} .
$$

Proof Let $D$ be an arbitrary orientation of $G$. Let $C, A$ and $B_{2}$ be as defined above, and let $u, v \in V(G)$. We bound $\theta(u, v)$ from above.

First assume that $u v \in E(G)$. We prove that

$$
\theta(u, v) \leq\left\{\begin{array}{l}
5, \text { if } u v \text { is a chord; } \\
3, \text { if } u v \text { is an outer edge }
\end{array}\right.
$$

If $u v$ is a chord, then $u$ and $v$ have two common neighbours, say $a$ and $b$. Every path between $u$ and $v$ in $G$, and thus in $D$, contains either $a$ or $b$ or the edge $u v$. Hence, apart from the path consisting of the edge $u v$, there exist at most two internally disjoint directed $u-v$ paths in $D$, and at most two internally disjoint directed $v-u$ paths in $D$. Hence, if $u v$ is a chord, then $\theta(u, v) \leq 5$.

If $u v$ is an outer edge, then $u$ and $v$ have exactly one common neighbour, say $a$. Every path between $u$ and $v$ in $G$ contains either $a$ or the edge $u v$. Hence, apart from the path consisting of the edge $u v$, there exist no two internally disjoint directed $u-v$ paths in $D$, and no two internally disjoint directed $v-u$ paths in $D$. Hence, if $u v$ is an outer edge, then $\theta(u, v) \leq 3$.

Now assume that $u v \notin E(G)$. We prove that

$$
\theta(u, v) \leq\left\{\begin{array}{l}
2, \text { if }\{u, v\} \cap B_{2} \neq \emptyset \\
4, \text { if }\{u, v\} \subseteq A \\
3, \text { otherwise. }
\end{array}\right.
$$

If $\{u, v\} \cap B_{2} \neq \emptyset$, then $\theta(u, v) \leq \min \left\{\operatorname{deg}_{G}(u), \operatorname{deg}_{G}(v)\right\}=2$. So assume that $\{u, v\} \cap B_{2}=\emptyset$. Clearly, since $u$ and $v$ are nonadjacent, and since $G$ is maximal outerplanar, there exist two adjacent vertices $a$ and $b$ of $G$ that separate $u$ and $v$. Hence, there exist at most two internally disjoint directed $u-v$ paths in $D$, and at most two internally disjoint directed $v-u$ paths in $D$. It follows that $\theta(u, v) \leq 4$. In order to complete the proof of (7), it suffices to show the following:

If $\theta(u, v)=4$, then $u$ is on a 4-cycle whose edges are chords.

Assume that $\theta(u, v)=4$. We may assume that if $C$ is traversed in clockwise direction, then $u, a$ and $b$ appear in this order. Let $u_{1}, u_{2}, \ldots, u_{k}$ be the neighbours of $u$ in clockwise order, where $u_{1}$ and $u_{k}$ are the neighbours of $u$ in $C$. Since $G$ is outerplanar, there exists $j$ such that $u_{1}, \ldots, u_{j}$ are in the $u-a$ subpath, and $u_{j+1}, \ldots, u_{k}$ are on the $b-u$ subpath of $C$. Then $\left\{u_{j}, u_{j+1}\right\}$ separates $u$ and $v$ in $G$. Also, $u u_{j}$ is a chord of $G$, since otherwise, if $u u_{j}$ was an outer edge, then every $u-v$ path in $G$ passes either through $u u_{j}$ or through $u_{j+1}$, implying that $\theta(u, v) \leq 3$. Similarly, $u u_{j+1}$ is a chord.

There exists a common neighbour $c$ of $u_{j}$ and $u_{j+1}$ distinct from $u$. We show that $u_{j} c$ is a chord of $G$. Suppose to the contrary that $u_{j} c$ is an outer edge of $G$. Since 
every $u-v$ path in $G$ passes through $\left\{u_{j}, u_{j+1}\right\}$, it follows that every $u-v$ path in $G$ passes either through the edge $u_{j} c$ or the vertex $u_{j+1}$, which implies that $\theta(u, v) \leq 3$, a contradiction. Hence $u_{j} c$ is a chord. Similarly we show that $u_{j+1} c$ is a chord. We conclude that $u, u_{j}, c, u_{j+1}, u$ is a 4-cycle whose edges are chords, so $u \in A$, and (8) follows.

We use (6) and (7) to bound the total connectivity of $D$. Let $x=|A|$ and $y=\left|B_{2}\right|$. First note that $G$ has $n$ unordered pairs $\{u, v\}$ of vertices that are joined by an outer edge. Of these, exactly $2 y$ pairs involve a vertex of degree 2 , so that $\theta(u, v) \leq 2$ in this case, and the remaining $n-2 y$ pairs satisfy $\theta(u, v) \leq 3$. Next, note that $G$ has $n-3$ pairs $\{u, v\}$ of vertices that are joined by a chord, and for these we have $\theta(u, v) \leq 5$.

Of the $\left(\begin{array}{l}n \\ 2\end{array}\right)-2 n+3$ pairs $\{u, v\}$ of nonadjacent vertices, at most $\left(\begin{array}{l}x \\ 2\end{array}\right)$ are contained in $A$, so $\theta(u, v) \leq 4$ for these pairs. There are $\left(\begin{array}{c}n \\ 2\end{array}\right)-\left(\begin{array}{c}n-y \\ 2\end{array}\right)$ unordered pairs $\{u, v\}$ of vertices involving a vertex of degree 2 , and $2 y$ of these are joined by an outer edge, while none of them are joined by chords. Hence there are $\left(\begin{array}{l}n \\ 2\end{array}\right)-\left(\begin{array}{c}n-y \\ 2\end{array}\right)-2 y$ pairs of nonadjacent vertices involving a vertex of degree 2 , so that $\theta(u, v) \leq 2$. The remaining $\left(\begin{array}{c}n-y \\ 2\end{array}\right)-\left(\begin{array}{l}x \\ 2\end{array}\right)-2 n+3+2 y$ pairs satisfy $\theta(u, v) \leq 3$. Summation of $\theta(u, v)$ over all unordered pairs $\{u, v\}$ yields that

$$
\sum_{\{u, v\} \subseteq V(G)} \theta(u, v) \leq 2\left(\begin{array}{l}
n \\
2
\end{array}\right)+\left(\begin{array}{c}
n-y \\
2
\end{array}\right)+\left(\begin{array}{l}
x \\
2
\end{array}\right)+2 n-6 .
$$

Now $y \geq \frac{1}{2} x+2$ by Lemma 5.2, hence

$$
\begin{aligned}
\sum_{\{u, v\} \subseteq V(G)} \theta(u, v) & \leq 2\left(\begin{array}{l}
n \\
2
\end{array}\right)+\left(\begin{array}{c}
n-2-\frac{1}{2} x \\
2
\end{array}\right)+\left(\begin{array}{l}
x \\
2
\end{array}\right)+2 n-6 \\
& =\frac{3}{2} n^{2}-\frac{1}{2} n-5-\frac{1}{2} n x+\frac{5}{8} x^{2}-\frac{5}{4} x
\end{aligned}
$$

Since $y \geq \frac{1}{2} x+2$, we have $n \geq x+y \geq \frac{3}{2} x+2$, and thus $x \leq \frac{2}{3} n-\frac{4}{3}$. Elementary calculus shows that the right hand side of (9), as a function of $x$, is maximized subject to $0 \leq x \leq \frac{2}{3} n-\frac{4}{3}$ when $x=0$. Substituting this yields

$$
\sum_{\{u, v\} \subseteq V(G)} \theta(u, v) \leq \frac{3}{2} n^{2}-\frac{1}{2} n-5
$$

and dividing by $n(n-1)$ yields the theorem.

The bound of Theorem 5.3 is asymptotically sharp. Let $G_{2 n}$ be the maximal outerplanar graph obtained from the path $P_{2 n}: v_{1} v_{2} \ldots v_{2 n}$ by adding the edges of the paths $Q: v_{1} v_{3} \ldots v_{2 n-1}$ and $R: v_{2} v_{4} \ldots v_{2 n}$. Note that $G_{2 n}$ is the square of the path of order $2 n$. Let $D_{2 n}$ be the orientation of $G_{2 n}$ obtained by directing the edges of $P_{2 n}$ from $v_{1}$ to $v_{2 n}$, the edges of $Q$ from $v_{2 n-1}$ to $v_{1}$, and the edges of $R$ from $v_{2 n}$ to $v_{2}$ (see Fig. 6). It is easy to see that all unordered pairs $\{u, v\}$ of vertices of $D_{2 n}$ satisfy 
Fig. 6 The orientation $D_{2 n}$ of maximal outerplanar graph $G_{2 n}$

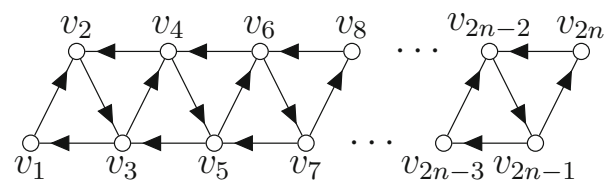

Table 1 The minimum value of $\bar{\kappa}_{\text {max }}$ over all maximal outerplanar graphs of a given order

\begin{tabular}{ll}
\hline Order & Minimum value of $\bar{\kappa}_{\max }$ \\
\hline 3 & 1 \\
4 & $13 / 12$ \\
5 & $23 / 20$ \\
6 & $7 / 6$ \\
7 & $25 / 21$ \\
8 & $67 / 56$ \\
9 & $29 / 24$ \\
\hline
\end{tabular}

$\theta(u, v)=3$, except for those $4 n-3$ pairs that involve a vertex of degree 2 in $G$, for which we have $\theta(u, v)=2$. Hence, we have $\bar{\kappa}_{\max }\left(G_{2 n}\right) \geq \bar{\kappa}\left(D_{2 n}\right)=\frac{3}{2}-\frac{4 n-3}{2 n(2 n-1)}$.

We now discuss a lower bound for $\bar{\kappa}_{\max }(G)$ if $G$ is a maximal outerplanar graph. Since maximal outerplanar graphs of order at least 3 are 2-connected, it follows immediately, from Robbins' Theorem, that $\bar{\kappa}_{\max }(G) \geq 1$ for every maximal outerplanar graph $G$ of order at least 3 . Moreover, this bound is tight since $\bar{\kappa}_{\max }\left(K_{3}\right)=1$. However, the graph $K_{3}$ seems exceptional.

Table 1 gives the minimum value of $\bar{\kappa}_{\max }(G)$ taken over all maximal outerplanar graphs $G$ of order $n$ for $3 \leq n \leq 9$. For $3 \leq n \leq 8$, the fan $F_{n}$ (the join of $K_{1}$ and $\left.P_{n-1}\right)$ is the unique maximal outerplanar graph of order $n$ that realizes this minimum value. For $n=9$, the fan $F_{9}$ and one other graph attain the minimum value of $\bar{\kappa}_{\text {max }}$. We do not pursue the details, but it appears that $\lim _{n \rightarrow \infty} \bar{\kappa}_{\text {max }}\left(F_{n}\right)=\frac{5}{4}$.

Based on the information in Table 1 , one might initially guess that the minimum value of $\bar{\kappa}_{\max }$ must increase with the order. However, we now describe an infinite family of maximal outerplanar graphs for which $\bar{\kappa}_{\max }$ is asymptotically at most $\frac{19}{18}+o(1)$. We conjecture that $\bar{\kappa}_{\max }(G) \geq 19 / 18$ for every maximal outerplanar graph $G$ of order at least 4 . The following example was inspired by the example from Henning and Oellermann (2004) that demonstrates sharpness for the lower bound of Theorem 1.3.

Example 5.4 Define a trigon as a triangle with every edge coloured red. Define a lozenge as a $K_{4}-e$ in which the edges of a perfect matching are coloured red, and all other edges are coloured black. Construct graph $G_{0}$ as follows: start with a trigon, and glue a lozenge red-on-red to every edge of the trigon, so that each vertex of the trigon has degree 5 in the resulting graph. See Fig. 7 for illustrations of a trigon, a lozenge, and the graph $G_{0}$.

Suppose $G_{i-1}$ has been constructed for some $i>0$. Construct $G_{i}$ from $G_{i-1}$ as follows: glue a trigon to every red outer edge of $G_{i-1}$, and then glue two lozenges (red-on-red) onto the two red outer edges of each trigon, so that each vertex in the trigon has degree 5 in the resulting graph (see Fig. 8a). 


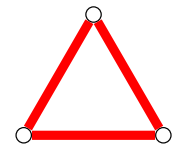

(a) A trigon

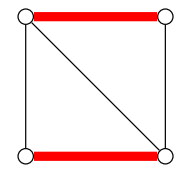

(b) A lozenge

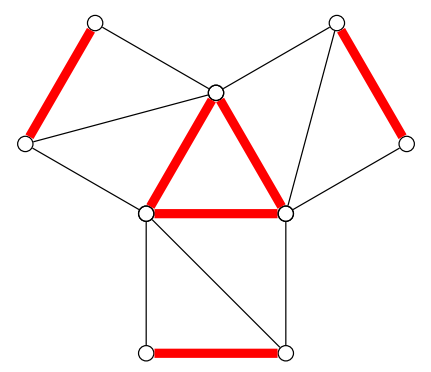

(c) The graph $G_{0}$

Fig. 7 A trigon, a lozenge, and the graph $G_{0}$

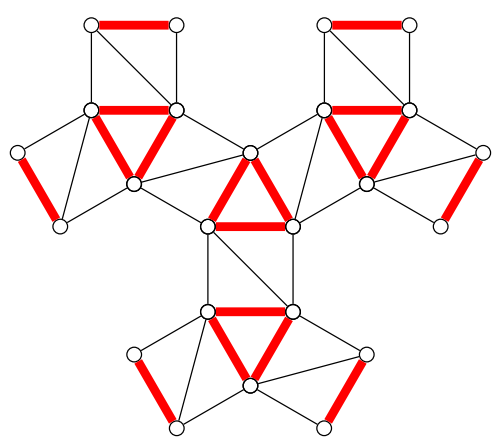

(a) The graph $G_{1}$

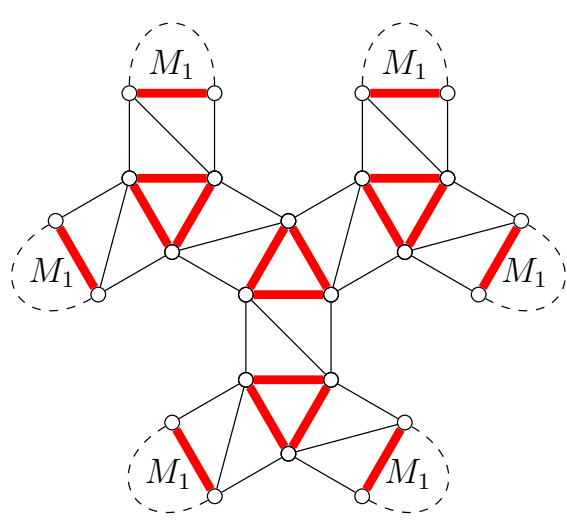

(b) The graph $H_{1}$

Fig. 8 The graphs $G_{1}$ and $H_{1}$

Now let $M_{i}$ be a sufficiently large maximal outerplanar graph with half of its vertices having degree 2 . For a given integer $k$, one can obtain such a graph $M_{i}$ of order $2 k$ from an arbitrary maximal outerplanar graph $F$ of order $k$ as follows. For every outer edge $e$ of $F$, add a new vertex $v_{e}$, and join $v_{e}$ to the endvertices of $e$. Colour one outer edge of $M_{i}$ red, and colour all other edges of $M_{i}$ black. Construct the graph $H_{i}$ from $G_{i}$ by gluing a copy of $M_{i}$ (red-on-red) to every outer red edge of $G_{i}$ (see Fig. 8b). We choose the graph $M_{i}$ in such a way that $\left|V\left(G_{i}\right)\right|=o\left(\left|V\left(M_{i}\right)\right|\right)$. This way, if one chooses two vertices $u$ and $v$ at random from $H_{i}$, then almost surely neither belongs to $G_{i}$, and for sufficiently large $i$, they are in fact almost surely in different copies of $M_{i}$.

Whenever a trigon and a lozenge (or a lozenge and a copy of $M_{i}$ ) share a red edge in $H_{i}$, we say that they are adjacent. If two vertices $u$ and $v$ of $H_{i}$ belong to distinct components of $H_{i}-E$, where $E$ is the set of black edges of a lozenge, then this lozenge is called a connector lozenge for $u$ and $v$.

We now consider an arbitrary orientation $D_{i}$ of $H_{i}$ for some $i \geq 0$. Suppose that vertices $u$ and $v$ are not in $G_{i}$, and are in distinct copies of $M_{i}$ (which is the case for almost all pairs of vertices asymptotically). In this case, there must be at least two 
Fig. 9 An orientation of the black edges of the lozenges adjacent to a trigon

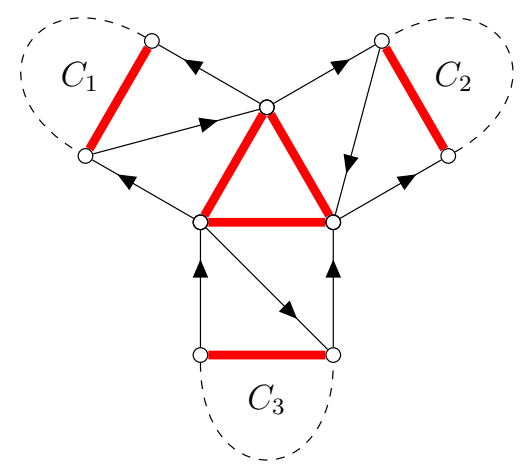

connector lozenges for $u$ and $v$, so $\theta(u, v) \leq 3$. Suppose that $\theta(u, v)=3$. We may assume, without loss of generality, that $\kappa(u, v)=2$ and $\kappa(v, u)=1$. So in every connector lozenge for $u$ and $v$, the independent black edges must be oriented away from $u$ towards $v$, and the third black edge must be oriented away from $v$ towards $u$. In particular, a majority of the black edges in any connector lozenge between $u$ and $v$ must be oriented away from $u$ towards $v$.

We take a step back to offer some intuition at this point. The deletion of a trigon from $H_{i}$ leaves three components $C_{1}, C_{2}$ and $C_{3}$. By the argument of the previous paragraph, there must be some pair of components, say $C_{1}$ and $C_{2}$, such that for every vertex $u$ in $C_{1}$ and every vertex $v$ in $C_{2}$, we have $\theta(u, v) \leq 2$ in $D_{i}$. Figure 9 shows an orientation of the black edges of the lozenges adjacent to a trigon. This orientation allows $\theta(u, v)=3$ for some $u \in V\left(C_{i}\right)$ where $i \in\{1,2\}$ and $v \in V\left(C_{3}\right)$. However, this forces $\theta(u, v)=2$ for every $u \in V\left(C_{1}\right)$ and $v \in V\left(C_{2}\right)$. Most importantly, this happens at the initial trigon of our construction, and this means that a large proportion of pairs will have $\theta(u, v) \leq 2$.

Define an auxiliary graph $A_{i}$ from $H_{i}$ as follows. The vertex set of $A_{i}$ is the set of trigons of $H_{i}$ together with the set of copies of $M_{i}$ as subgraphs in $H_{i}$. Two vertices of $A_{i}$ are adjacent in $A_{i}$ if and only if they are adjacent to a common lozenge in $H_{i}$. (So edges in $A_{i}$ correspond exactly to lozenges in $H_{i}$.) The orientation $D_{i}$ of $H_{i}$ gives rise to an orientation $D_{i}^{*}$ of $A_{i}$ as follows: orient edge $u v$ in $A_{i}$ as $(u, v)$ if a majority of the black arcs in the corresponding lozenge of $H_{i}$ are directed away from $u$ towards $v$.

In order to bound the average connectivity of $D_{i}$, we now bound the average connectivity between leaves in the orientation $D_{i}^{*}$ of $A_{i}$. There are $3 \cdot 2^{i}$ leaves in $A_{i}$, and by a straightforward induction, one can prove that there are at most $2 \cdot 4^{i}$ pairs of leaves in $D_{i}^{*}$ that are connected by a directed path. Thus, if we pick two leaves of $D_{i}^{*}$ at random, then the probability that there is a directed path between them (and hence the probability that the connector lozenges between the corresponding copies of $M_{i}$ all have a majority of black edges oriented the same way) tends to at most $p=4 / 9$.

We now return to the orientation $D_{i}$ of $H_{i}$. For each copy of $M_{i}$, we consider the set of vertices in $M_{i}$ that don't belong to $G_{i}$ (i.e., those that are not incident with the red edge). Note that exactly half of these vertices have degree 2 , and hence the proportion of these vertices that can have $\theta$ value 3 with some vertex in another copy of $M_{i}$ is at 
most $q=1 / 2$. Thus, the average connectivity of $D_{i}$ is at most

$$
\frac{3 p q^{2}+2\left(1-p q^{2}\right)}{2}+o(1)=1+\frac{p q^{2}}{2}+o(1) \leq 1+\frac{1}{18}+o(1)
$$

Since $D_{i}$ was an arbitrary orientation of $H_{i}$, we have

$$
\bar{\kappa}_{\max }\left(H_{i}\right) \leq 1+\frac{1}{18}+o(1) .
$$

Note that we have only shown an upper bound on $\bar{\kappa}_{\max }\left(H_{i}\right)$; i.e., we have not shown that $\bar{\kappa}_{\max }\left(H_{i}\right) \geq 1+\frac{1}{18}$. However, for each $i$, we can choose $M_{i}$ so that we do in fact have

$$
\lim _{i \rightarrow \infty} \bar{\kappa}_{\max }\left(H_{i}\right)=1+\frac{1}{18}
$$

We omit the details of explicitly describing both $M_{i}$ and an orientation of the resulting graph $H_{i}$, and then demonstrating a lower bound on the average connectivity of this orientation. However, we note that an optimal orientation of $A_{i}$, which also maximizes the average connectivity between leaves, is easily obtained using the results of Henning and Oellermann (2004). An orientation of the black edges in the lozenges of $H_{i}$ can be "lifted" from this optimal orientation of $A_{i}$ as follows: if $(u, v)$ is an arc in $A_{i}$, then orient the independent black edges in the corresponding lozenge of $H_{i}$ away from $u$ towards $v$, and orient the last black edge in the corresponding lozenge of $H_{i}$ away from $v$ towards $u$. This orientation of the lozenges more or less determines the orientations of the edges in the trigons.

\subsection{Orientations of 2-trees}

We present a lower bound for $\bar{\kappa}_{\text {max }}(G)$ if $G$ is a 2-tree.

Theorem 5.5 Let $G$ be a 2-tree of order $n \geq 3$. Then

$$
\bar{\kappa}_{\max }(G) \geq 1+\frac{n-3}{n(n-1)},
$$

and this bound is sharp for all $n$.

Proof We show by induction on $n$ that every 2-tree $G$ of order $n$ has a strong orientation $D$ with $K(D) \geq n^{2}-3$. If $n=3$, then $G$ is a triangle, and orienting the edges of $K_{3}$ as a directed cycle yields a digraph of total connectivity 6 , so the statement holds for $n=3$. Now let $G$ be a 2 -tree of order $n$. Then $G$ has a vertex $u$ such that $G-u$ is a 2-tree, and the neighbourhood of $u$ in $G$ consists of two adjacent vertices, $v$ and $w$ say. By our induction hypothesis, $G-u$ has a strong orientation $D^{\prime}$ of total connectivity at least $(n-1)^{2}-3$. We extend $D^{\prime}$ to a strong orientation $D$ of $G$ by orienting the edges $u v$ and $u w$ so that they form a directed 3-cycle together with $v w$. Then $D$ is strong. 
Hence $D$ contains $2(n-1)$ paths, from $u$ to and from all vertices in $D^{\prime}$. Furthermore, $D$ contains a new path between $v$ and $w$ through $u$, that has no edges in common with any path in $D^{\prime}$. Hence $K(D) \geq K\left(D^{\prime}\right)+2 n-1 \geq(n-1)^{2}-3+2 n-1=n^{2}-3$.

For a given $n \geq 3$, let $G_{n}=K_{2}+\bar{K}_{n-2}$ (the join of $K_{2}$ and $\bar{K}_{n-2}$ ) and let $u, v$ be the vertices of degree $n-1$ in $G_{n}$. Let $D_{n}$ be an optimal orientation of $G_{n}$. If $a, b$ is a pair of vertices of $G_{n}$ such that $\{a, b\} \neq\{u, v\}$, then $\theta(a, b) \leq 2$. Moreover, $\theta(u, v) \leq n-1$. Hence $K\left(D_{n}\right) \leq n(n-1)+n-3$ and thus $\bar{\kappa}_{\max }\left(G_{n}\right) \leq 1+\frac{n-3}{n(n-1)}$. Thus the given bound is sharp.

We also conjecture that if $G$ is a 2-tree of order $n$ for which $\bar{\kappa}_{\max }(G)$ is largest, then $G$ is maximal outerplanar. If this conjecture holds, then the results of Sect. 5.1 tell us that $\bar{\kappa}_{\max }(G) \leq \frac{3}{2}+o(1)$ for every 2 -tree $G$, and that this bound is asymptotically sharp.

\section{Conclusion}

The problem of finding the maximum average connectivity among all orientations of a graph was introduced in Henning and Oellermann (2004), where the following asymptotically tight bounds for the maximum average connectivity of a tree $T$ were established:

$$
\frac{2}{9}<\bar{\kappa}_{\max }(T) \leq \frac{1}{2}
$$

In this paper we focused on finding bounds for $\bar{\kappa}_{\text {max }}(G)$ and $\bar{\kappa}_{\max }(G) / \bar{\kappa}(G)$ for graphs $G$ belonging to certain classes that extend trees.

We showed that if $G$ is a minimally 2 -connected graph, then

$$
1 \leq \bar{\kappa}_{\max }(G)<\frac{5}{4}
$$

and

$$
\frac{4}{9}<\frac{\bar{\kappa}_{\max }(G)}{\bar{\kappa}(G)}<\frac{5}{8}
$$

We know that the lower bound for $\bar{\kappa}_{\max }(G)$ is sharp, but suspect that the upper bound can be improved.

Problem 6.1 Find an asymptotically sharp upper bound for $\bar{\kappa}_{\max }(G)$ if $G$ is a minimally 2-connected graph.

The bounds on the ratio have not yet been shown to be tight.

Problem 6.2 Determine asymptotically sharp upper and lower bounds for the ratio $\frac{\bar{\kappa}_{\max }(G)}{\bar{\kappa}(G)}$ if $G$ is a minimally 2 -connected graph. 
If the upper bound of $5 / 4$ for $\bar{\kappa}_{\max }(G)$ can be improved, this will also give rise to an improved upper bound for the ratio $\frac{\bar{\kappa}_{\max }(G)}{\bar{\kappa}(G)}$ of minimally 2-connected graphs $G$.

For every maximal outerplanar graph $G$, we proved that

$$
\bar{\kappa}_{\max }(G) \leq \frac{3}{2}+o(1)
$$

and that this bound is asymptotically sharp. For the lower bound, we propose the following.

Conjecture 6.3 For every maximal outerplanar graph $G$ of order at least 4, we have

$$
\bar{\kappa}_{\max }(G) \geq 19 / 18 \text {. }
$$

We demonstrated that if this conjectured bound holds, then it is asymptotically sharp.

For a graph $G$ in the class of 2-trees (which contain the maximal outerplanar graphs), we showed that the bound $\bar{\kappa}_{\max }(G) \geq 1$ is asymptotically sharp. We propose the following conjecture.

Conjecture 6.4 If $G$ is a 2-tree of order $n \geq 3$ for which $\bar{\kappa}_{\max }(G)$ is largest, then $G$ is maximal outerplanar.

If this conjecture holds, then the upper bound given in Theorem 5.3 is also an upper bound for $\bar{\kappa}_{\max }(G)$ if $G$ is a 2 -tree.

We observed that if $G$ is a graph, then $\bar{\kappa}_{\max }(G) / \bar{\kappa}(G) \leq 1$, and we proved that this bound is asymptotically sharp. However, the following remains open.

Problem 6.5 Does there exist a constant $c>0$ such that $\bar{\kappa}_{\max }(G) / \bar{\kappa}(G) \geq c$ for every (2-)connected graph $G$ ?

For every tree $T$, it is known that $\bar{\kappa}_{\max }(T) / \bar{\kappa}(T)>2 / 9$, and that this bound is asymptotically sharp. For every 3 -connected cubic graph $G$, the fact that $\bar{\kappa}_{\max }(G) / \bar{\kappa}(G) \geq$ $1 / 3$ follows from Robbins' Theorem, and we demonstrated that this bound is asymptotically sharp.

Very little is known about the global connectivity of optimal orientations of graphs. In particular, it would be interesting if the following could be answered.

Problem 6.6 Is every optimal orientation of every 2-(edge-)connected graph strongly connected?

Even the following weaker version of this problem remains open.

Problem 6.7 Does every 2-(edge-)connected graph have a strong optimal orientation?

Acknowledgements The authors wish to thank the Banff International Research Station for their support of the focussed research group number 18frg233, "Measuring the Connectedness of Graphs and Digraphs", during which most of the research for this manuscript was completed. 


\section{References}

Beineke LW, Oellermann OR, Pippert RE (2002) The average connectivity of a graph. Discrete Math 252:31-45

Bollobás B (2004) Extremal graph theory. Dover Publications, Mineola

Casablanca RM, Mol L, Oellermann OR (2021) Average connectivity of minimally 2-connected graphs and average edge-connectivity of minimally 2-edge-connected graphs. Discrete Appl Math 289:233-247

Chvátal V (1973) Tough graphs and Hamiltonian circuits. Discrete Math 5:215-228

Dankelmann P, Oellermann OR (2003) On the average connectivity of a graph. Discrete Appl Math 129:305318

Dirac GA (1967) Minimally 2-connected graphs. J Reine Angew Math 228:204-216

Durand de Gevigney O (2020) On Frank's conjecture on $k$-connected orientations. J Combin Theory Ser B 141:105-114

Henning MA, Oellermann OR (2004) The average connectivity of a digraph. Discrete Appl Math 140:143153

Henning MA, Oellermann OR (2001) The average connectivity of regular multipartite tournaments. Australas J Combin 23:101-113

Mader W (1972) Ecken vom Grad $n$ in minimalen $n$-fach zusammenhängenden Graphen. Arch Math 23:219-224

Mader W (1978) A reduction method for edge-connectivity in graphs. Ann Discrete Math 3:145-164

Nash-Williams CSJA (1960) On orientations, connectivity and odd-vertex-pairings in finite graphs. Can J Math 12:555-567

Oellermann OR (2013) Menger's theorem. In: Beineke LW, Wilson RJ (eds) Topics in structural graph theory. Cambridge University Press, Cambridge

Plummer MD (1968) On minimal blocks. Trans. Am. Math. Soc. 134(1):85-94

Robbins HE (1939) Questions, discussions, and notes: a theorem on graphs, with an application to a problem of traffic control. Am. Math. Mon. 46:281-283

Thomassen C (1989) Configurations in graphs of large minimum degree, connectivity, or chromatic number. In: Proceedings of the third international conference on combinatorial mathematics, New York 1985, Annals of the New York Academy of Sciences, New York, vol 555, pp 402-412

Thomassen C (2014) Strongly 2-connected orientations of graphs. J Combin Theory Ser B 110:67-78

Publisher's Note Springer Nature remains neutral with regard to jurisdictional claims in published maps and institutional affiliations. 